\title{
Interstellar polarization and extinction towards the young open cluster NGC 1502
}

\author{
G. A. Topasna ${ }^{1}$, N. T. Kaltcheva ${ }^{2}$, and E. Paunzen ${ }^{3}$ \\ ${ }^{1}$ Department of Physics and Astronomy, Virginia Military Institute, Lexington, VA 24450, USA \\ e-mail: TopasnaGA@vmi .edu \\ 2 Department of Physics and Astronomy, University of Wisconsin Oshkosh, 800 Algoma Blvd., Oshkosh, WI 54901, USA \\ e-mail: kaltchev@uwosh.edu \\ ${ }^{3}$ Department of Theoretical Physics and Astrophysics, Masaryk University, Kotlářská 2, 61137 Brno, Czech Republic \\ e-mail: epaunzen@physics.muni.cz
}

Received 6 September 2017 / Accepted 6 March 2018

\begin{abstract}
Aims. NGC 1502 is located at the periphery of the Cam OB1 association and probably within the Orion Spur, with published distance estimates varying between 0.7 and $1.5 \mathrm{kpc}$. We combine new polarimetric observations and existing $u v b y \beta$ and $U B V$ photometries to provide new estimates of the cluster's parameters.

Methods. We present new multi-wavelength polarization observations of 22 stars in the direction of the cluster and, using the wavelength of maximum polarization, calculate the total-to-selective extinction ratio for each of these stars. These are combined with homogenized $u v b y \beta$ and $U B V$ photometries to obtain the individual stellar distances and study the structure of the field.

Results. We found no evidence of significant extinction variation across the cluster and obtained an average color excess $E(b-y)=0.56 \pm 0.02 \mathrm{mag}$, corresponding to $E(B-V)=0.76$ mag. Given the uniformity of both $p_{\max }$ and $\lambda_{\max }$ toward the cluster and the distribution of the color excess with distance, it seems likely that the polarization in this region arises from aligned dust grains in the foreground of the cluster, not farther than 500-600 pc. Based on 20 stars located beyond 500 pc within the studied field of view, we obtained an average polarization-based total-to-selective extinction ratio $R=2.83 \pm 0.14$ yielding visual absorption $A_{V}=2.14 \pm 0.16 \mathrm{mag}$. Using 11 stars with $u v b y \beta$ data that represent the main sequence of the cluster, we calculated a photometric distance of $1117_{-89}^{+96} \mathrm{pc}$. This estimate is fully supported by the $U B V$-based analysis and the new individual membership probabilities that we derive. Assuming a solar metallicity yields an age of 5 Myr. Our analysis yields controversial results regarding the membership of the stars associated with the SZ Cam system (HD 25638 and HD 25639) to the cluster.
\end{abstract}

Key words. stars: early type - open clusters and associations: individual: NGC 1502

\section{Introduction}

NGC 1502 is a moderately reddened open cluster located at $l=143.6^{\circ}, b=+7.6^{\circ}$, near the periphery of the Cam OB1 association. It is a very young cluster, with an almost vertical main sequence. It could be an example of a disrupting or expanding unstable cluster, with a large size and a small core (see recent investigation by Alves et al. 2012). The cluster contains the earlytype eclipsing binary SZ Cam (HD 25638), which is one of the components of the visual double star representing the brightest members of the cluster (Lorenz et al. 1998; Hohle et al. 2009).

The initial investigations of NGC 1502 probably date back to Hoag (1953) who obtained polarization and photoelectric photometry of stars in five open clusters, including NGC 1502. He found that the ratio of polarization to reddening is a constant for each cluster, but varies with the galactic longitude of the clusters. The cluster is included in the $U B V$ catalog by Hoag et al. (1961). Since then, NGC 1502 has been investigated by various photometric means, as summarized by Paunzen et al. (2005). While the estimates of the average color excess $E(B-V)$ provided by different authors are fairly consistent (between 0.7 and 0.78 ), the values of the total-to-selective extinction $R$, and hence the true distance modulus and the cluster age estimates differ significantly. The first distance estimates come from
Johnson et al. (1961; 870 pc) and Purgathofer (1961; 955 pc, $R$ $=3$, total extinction $A_{V}=2.1 \mathrm{mag}$ ). Combining $U B V$ and $u v b y$ photometries, Reimann \& Pfau (1987) obtained 960 pc $(R=$ 3.14) and an age of 11 Myr. Based on $J H K$ and $u v b y \beta$ photometries, Tapia et al. (1991) estimated abnormal $R=2.42 \pm 0.09$, a distance of $1.5 \mathrm{kpc}\left(A_{V}=1.83 \pm 0.03 \mathrm{mag}\right)$ and an age of 5 Myr. Delgado et al. (1992) obtained new $u v b y \beta$ photometry of 22 stars in the cluster and a distance of 929 pc (for an accepted $R=3.18$ ). In order to address concerns related to the choice of reddened standard stars, Crawford (1994b) performed new uvby $\beta$ photometry, revising the distance to $875 \mathrm{pc}$.

More recently, Hohle et al. (2009) used BVRI photometry and stellar evolution models to calculate $A_{V}$ and based on six cluster members with known spectral types obtained $2.09 \mathrm{mag}$. Using the narrow band three-filter $\Delta \alpha$ photometric system, Paunzen et al. (2005) estimated a distance of $1080 \mathrm{pc}$ and an age of $7.9 \mathrm{Myr}$ (accepting $R=2.57$ ). Based on 2MASS color-magnitude diagrams, Alves et al. (2012) obtained a distance of $1259 \mathrm{pc}$ and an age of $5 \mathrm{Myr}$ (for $R=3.1, E(B-V)=$ $0.6 \pm 0.1)$. However, as discussed by the latter authors, this larger distance should be viewed in light of the still present disagreement between physical parameters based on near infrared data and optical and UV data. Johnson $U B V$ and Cousins $R I$ CCD photometry down to 22 mag was recently obtained by Tripathi et al. (2013), yielding a distance of $1.0 \pm 0.1 \mathrm{kpc}$ 
(for $R=3.1$ ), an average $E(B-V)=0.68 \pm 0.05$, and an age of $10.0 \pm 0.1 \mathrm{Myr}$.

Polarmetric observations of cluster stars and field stars in the direction of NGC 1502 are provided by many authors (see Weitenbeck et al. (1996, 2008) for complete reviews). These measurements showed that the maximum degree of polarization was on the order of 5-6\% with polarization vectors aligned in the direction of the Galactic plane.

With an aim to address the inconsistencies in the distance and extinction determination, we present new multi-wavelength polarization measurements of 22 stars in the direction of the cluster. We use the wavelength of maximum polarization to calculate the total-to-selective extinction ratio for each of these stars and also an average value for the cluster. Further, we combine these estimates with $u v b y \beta$ and $U B V$ photometric data to obtain individual stellar distances and provide a more detailed stellar distribution of this region.

\section{Polarimetric observations and analysis}

UBVRI images of NGC 1502 were obtained on the night of 3 November 2015 with the field centered on HD 25638. The unpolarized standard star HD 20630 ( $\kappa$ Cet) (Serkowski 1974) was imaged to remove instrumental polarization. All images were obtained using the optical polarimeter on the $0.5 \mathrm{~m}, \mathrm{f} / 13.5$ Cassegrain telescope at the Virginia Military Institute Observatory located at McKethan Park outside of Lexington, Virginia. A description of the polarimeter and its operations is given by Topasna et al. (2013, 2017).

Aperture photometry was used to determine the ordinary and extraordinary flux $I^{o}$ and $I^{e}$ and, based on the method described by di Serego Alighieri (1997), the ratios

$R_{q}^{2}=\frac{I_{0^{\circ}}^{o}}{I_{0^{\circ}}^{e}} \frac{I_{45^{\circ}}^{e}}{I_{45^{\circ}}^{o}} ; \quad R_{u}^{2}=\frac{I_{22.5^{\circ}}^{o}}{I_{22.5^{\circ}}^{e}} \frac{I_{67.5^{\circ}}^{e}}{I_{67.5^{\circ}}^{o}}$,

were computed. The normalized Stokes parameters $q$ and $u$ were determined from the equations $q=\left(R_{q}-1\right) /\left(R_{q}+1\right)$ and $u=\left(R_{u}-1\right) /\left(R_{u}+1\right)$. The degree of polarization $p_{0}=\sqrt{q^{2}+u^{2}}$ and position angle $\theta=\frac{1}{2} \arctan \frac{u}{q}$ were computed along with their respective standard errors, $\sigma_{p}=\left[q^{2} \sigma_{q}^{2}+u^{2} \sigma_{u}^{2}\right]^{1 / 2} / p$ and $\sigma_{\theta}=\left[q^{2} \sigma_{u}^{2}+u^{2} \sigma_{q}^{2}\right]^{1 / 2} / 2 p^{2}$. The star's position angle $\theta$ is measured with respect to a line of constant right ascension with a positive angle increasing toward the east. Inherent bias in the degree of polarization was accounted for using the Wardle $\&$ Kronberg (1974) estimator $p=\sqrt{p_{0}^{2}-\sigma_{p}^{2}}$ as discussed by Simmons \& Stewart (1985). The broad-band polarization measurements for 22 bright stars in the direction of the cluster are listed in Table 1.

The wavelength dependence of polarization is described by the empirical relationship (Serkowski et al. 1975)

$p(\lambda) / p_{\max }=\exp \left[-K \ln ^{2}\left(\lambda_{\max } / \lambda\right)\right]$

where $p_{\max }$ is the maximum degree of polarization that occurs at the wavelength $\lambda_{\max }$ [see Clarke (2010), particularly Chap. 10]. The parameter $K$ was shown by Wilking et al. (1982) to be a function of $\lambda_{\max }$ given by $K=(-0.10 \pm 0.05)+(1.86 \pm 0.09) \lambda_{\max }$, the dependence generally being ascribed to grain size, distribution, and growth (Wilking et al. 1982, Aannestad 1982, Aannestad \& Greenberg 1983). However, the correlation between $K$ and $\lambda_{\max }$ was shown by Clarke \& Al-Roubaie (1983,
1984) to also depend on the signal-to-noise ratio, choice of bandpass, and the presence of clouds along the line of sight. Leaving $K$ to be a free-fit parameter does not significantly change the derived maximum degree of polarization or wavelength of maximum polarization; on the contrary, it actually leads to a better nonlinear fit to the Serkowski equation.

A nonlinear regression was fit to the Serkowski equation using the wavelength-dependent data for each star given in Table 1. The corresponding Serkowski fits are shown in Fig. 1 and the parameters $p_{\max }, \lambda_{\max }$, and $K$, along with their uncertainties and goodness of fit, are listed in Table 2. For star identification throughout this paper we use the WEBDA numbering system, which follow Purgathofer (1961) for the vast majority of the stars considered here. The standard polarized star, HD 7927 ( $\phi$ Cas) was also imaged and a nonlinear regression fit to the data is shown by the solid curve in Fig. 2 and has an $r^{2}$ of 0.993 . The maximum polarization is $3.62 \pm 0.03 \%$ and occurs at $0.520 \pm 0.006 \mu \mathrm{m}$ with $K$ equal to $1.40 \pm 0.11$ and a position angle of $(93.18 \pm 0.01)^{\circ}$. The original determination of the maximum degree of interstellar polarization for HD 7927 was obtained by Serkowski (1974) to be $3.4 \%$ at $\lambda_{\max }=0.51 \mu \mathrm{m}$ and $\theta\left(\lambda_{\max }\right)=94^{\circ}$. Later measurements by Hsu \& Breger (1982) found $p_{\max }=(3.41 \pm 0.02) \%$ at $\lambda_{\max }=(0.515 \pm 0.006) \mu \mathrm{m}$ and they list only the polarization angle in the $V$ filter, which they report as $(92.3 \pm 0.1)^{\circ}$.

The polarization vectors in the $V$-band for the 22 stars observed here are plotted in Fig. 3. The size of the symbols corresponds to the brightness of the stars. Open circles are used for the stars that are included in the first Gaia data release (Gaia Collaboration 2016). The length of each vector is proportional to the maximum degree of polarization of the corresponding star (a scale bar is included for reference). The dotted line delineates the Galactic latitude at $b=7.59^{\circ}$. The degree of polarization in the $V$-band is averaging around $6.9 \%$, with a maximum of $8.8 \%$ and minimum of $6.3 \%$. The polarization is fairly uniform across the region.

In Fig. 3 we see that the polarization vectors are closely aligned with the Galactic plane. When referenced to the Galactic plane, the average galactic polarization angle is $2.5 \pm 0.6$ degrees (although a median value of $\theta_{G}$ is 2.0 degrees). Here $\theta_{G}$ is the angle that the polarization vector makes with respect to the Galactic plane with a positive angle increasing toward the north Galactic pole. The average direction and dispersion we measure is consistent with the results presented in Figs. 5 and 9 of the Planck all-sky polarization maps (Planck Collaboration Int. XIX 2015). In particular their left-hand column in Fig. 9 shows an enlargement of the highly polarized region extending from $120^{\circ}$ to $160^{\circ}$ in Galactic longitude and $0^{\circ}$ to $20^{\circ}$ in Galactic latitude. NGC 1502 is located within this field. Smoothed to a resolution of $1^{\circ}$, the polarization fraction from dust emission at $353 \mathrm{GHz}$ shows position vectors that are generally parallel to the Galactic plane and display little dispersion. Likewise, the polarization exhibited by the dust is on the order of $12-14 \%$ at the location of NGC 1502. Presumably, the dust responsible for the emission seen in the integrated line of sight all-sky polarization map is also responsible for the optical polarization of starlight that we observe. However, the spatial resolution of the currently available Planck data is not sufficient for a more detailed conclusion.

Earliest polarization measurements of stars in the vicinity of NGC 1502 are from Hiltner (1951), Appenzeller (1968) and Serkowski et al. (1975) but are not specifically of the cluster itself. More recent polarization observations of NGC 1502 and surrounding stars are performed by Weitenbeck (1997, 2004) 
G. A. Topasna et al.: NGC 1502

Table 1. Polarimetric measurements of stars toward NGC 1502.

\begin{tabular}{|c|c|c|c|c|c|c|c|}
\hline Star & Filter & $p(\%)$ & $\theta$ & Star & Filter & $p(\%)$ & $\theta$ \\
\hline \multirow[t]{5}{*}{1} & $U$ & $5.807 \pm 0.052$ & $139.49 \pm 0.01$ & \multirow[t]{5}{*}{36} & $U$ & $6.772 \pm 0.030$ & $137.81 \pm 0.03$ \\
\hline & $B$ & $6.428 \pm 0.023$ & $141.58 \pm 0.01$ & & $B$ & $7.004 \pm 0.018$ & $142.45 \pm 0.02$ \\
\hline & $V$ & $6.416 \pm 0.021$ & $142.15 \pm 0.01$ & & $V$ & $6.781 \pm 0.121$ & $140.08 \pm 0.03$ \\
\hline & $R$ & $6.036 \pm 0.017$ & $142.41 \pm 0.01$ & & $R$ & $6.498 \pm 0.084$ & $141.78 \pm 0.02$ \\
\hline & $I$ & $4.944 \pm 0.027$ & $142.61 \pm 0.01$ & & $I$ & $5.683 \pm 0.095$ & $140.57 \pm 0.03$ \\
\hline \multirow[t]{5}{*}{2} & $U$ & $6.172 \pm 0.067$ & $141.28 \pm 0.01$ & \multirow[t]{5}{*}{37} & $U$ & $5.766 \pm 0.210$ & $142.41 \pm 0.04$ \\
\hline & $B$ & $6.666 \pm 0.026$ & $140.23 \pm 0.01$ & & $B$ & $6.446 \pm 0.118$ & $142.70 \pm 0.03$ \\
\hline & $V$ & $6.702 \pm 0.023$ & $140.69 \pm 0.01$ & & $V$ & $6.850 \pm 0.151$ & $141.64 \pm 0.04$ \\
\hline & $R$ & $6.207 \pm 0.017$ & $140.90 \pm 0.02$ & & $R$ & $6.572 \pm 0.126$ & $142.33 \pm 0.03$ \\
\hline & $I$ & $5.096 \pm 0.014$ & $140.81 \pm 0.01$ & & $I$ & $5.491 \pm 0.177$ & $140.75 \pm 0.05$ \\
\hline \multirow[t]{5}{*}{7} & $U$ & - & - & \multirow[t]{5}{*}{40} & $U$ & $6.494 \pm 0.566$ & $147.22 \pm 0.01$ \\
\hline & $B$ & $6.802 \pm 0.274$ & $140.34 \pm 0.06$ & & $B$ & $7.124 \pm 0.128$ & $141.75 \pm 0.03$ \\
\hline & $V$ & $6.792 \pm 0.128$ & $142.86 \pm 0.03$ & & V & $6.699 \pm 0.083$ & $143.31 \pm 0.02$ \\
\hline & $R$ & $6.321 \pm 0.091$ & $141.50 \pm 0.02$ & & $R$ & $6.629 \pm 0.080$ & $141.61 \pm 0.02$ \\
\hline & $I$ & $5.275 \pm 0.115$ & $140.63 \pm 0.03$ & & $I$ & $5.297 \pm 0.086$ & $141.60 \pm 0.03$ \\
\hline \multirow[t]{5}{*}{10} & $U$ & $6.695 \pm 0.266$ & $137.16 \pm 0.04$ & \multirow[t]{5}{*}{42} & $U$ & - & - \\
\hline & $B$ & $6.649 \pm 0.151$ & $137.66 \pm 0.04$ & & $B$ & $6.138 \pm 0.292$ & $143.11 \pm 0.07$ \\
\hline & $V$ & $6.563 \pm 0.097$ & $138.99 \pm 0.02$ & & $V$ & $6.316 \pm 0.101$ & $142.64 \pm 0.03$ \\
\hline & $R$ & $6.743 \pm 0.186$ & $139.46 \pm 0.04$ & & $R$ & $5.887 \pm 0.108$ & $142.23 \pm 0.03$ \\
\hline & $I$ & $5.260 \pm 0.201$ & $135.99 \pm 0.06$ & & $I$ & $4.891 \pm 0.134$ & $140.84 \pm 0.04$ \\
\hline \multirow[t]{5}{*}{11} & $U$ & $4.322 \pm 0.644$ & $141.60 \pm 0.17$ & \multirow[t]{5}{*}{43} & $U$ & $6.172 \pm 0.067$ & $141.28 \pm 0.01$ \\
\hline & $B$ & $6.025 \pm 0.131$ & $140.54 \pm 0.03$ & & $B$ & $7.019 \pm 0.119$ & $139.64 \pm 0.03$ \\
\hline & $V$ & $6.262 \pm 0.065$ & $139.54 \pm 0.02$ & & $V$ & $6.942 \pm 0.044$ & $141.76 \pm 0.01$ \\
\hline & $R$ & $5.954 \pm 0.068$ & $140.98 \pm 0.02$ & & $R$ & $6.483 \pm 0.037$ & $140.52 \pm 0.01$ \\
\hline & $I$ & $4.855 \pm 0.053$ & $140.61 \pm 0.02$ & & $I$ & $5.260 \pm 0.051$ & $140.81 \pm 0.02$ \\
\hline \multirow[t]{5}{*}{12} & $U$ & $5.872 \pm 0.080$ & $139.42 \pm 0.02$ & \multirow[t]{5}{*}{44} & $U$ & $4.581 \pm 0.591$ & $139.67 \pm 0.14$ \\
\hline & $B$ & $6.699 \pm 0.049$ & $139.92 \pm 0.01$ & & $B$ & $6.798 \pm 0.056$ & $138.24 \pm 0.01$ \\
\hline & $V$ & $6.750 \pm 0.023$ & $139.18 \pm 0.01$ & & $V$ & $6.578 \pm 0.046$ & $142.62 \pm 0.01$ \\
\hline & $R$ & $5.688 \pm 0.116$ & $140.72 \pm 0.03$ & & $R$ & $6.336 \pm 0.033$ & $142.59 \pm 0.01$ \\
\hline & $I$ & $5.202 \pm 0.028$ & $139.48 \pm 0.01$ & & $I$ & $5.037 \pm 0.032$ & $143.54 \pm 0.01$ \\
\hline \multirow[t]{5}{*}{16} & $U$ & - & - & \multirow[t]{5}{*}{45} & $U$ & - & - \\
\hline & $B$ & $5.964 \pm 0.103$ & $139.32 \pm 0.03$ & & $B$ & $5.918 \pm 0.341$ & $141.98 \pm 0.09$ \\
\hline & $V$ & $6.283 \pm 0.051$ & $138.42 \pm 0.01$ & & $V$ & $6.286 \pm 0.097$ & $137.40 \pm 0.02$ \\
\hline & $R$ & $5.922 \pm 0.047$ & $138.16 \pm 0.01$ & & $R$ & $5.907 \pm 0.052$ & $138.72 \pm 0.01$ \\
\hline & $I$ & $4.891 \pm 0.094$ & $134.07 \pm 0.03$ & & $I$ & $4.904 \pm 0.112$ & $139.62 \pm 0.04$ \\
\hline \multirow[t]{5}{*}{23} & $U$ & - & - & \multirow[t]{5}{*}{49} & $U$ & $5.993 \pm 0.581$ & $141.47 \pm 0.11$ \\
\hline & $B$ & $7.573 \pm 0.082$ & $137.81 \pm 0.02$ & & $B$ & $6.167 \pm 0.095$ & $142.13 \pm 0.02$ \\
\hline & $V$ & $7.745 \pm 0.051$ & $137.46 \pm 0.01$ & & V & $6.431 \pm 0.109$ & $139.45 \pm 0.03$ \\
\hline & $R$ & $7.750 \pm 0.036$ & $136.52 \pm 0.01$ & & $R$ & $6.079 \pm 0.073$ & $141.26 \pm 0.02$ \\
\hline & $I$ & $6.205 \pm 0.060$ & $137.99 \pm 0.02$ & & $I$ & $5.076 \pm 0.168$ & $138.14 \pm 0.05$ \\
\hline \multirow[t]{5}{*}{26} & $U$ & $6.779 \pm 0.141$ & $134.47 \pm 0.02$ & 52 & $U$ & - & - \\
\hline & $B$ & $7.886 \pm 0.085$ & $136.27 \pm 0.02$ & & $B$ & $5.639 \pm 0.295$ & $141.27 \pm 0.08$ \\
\hline & $V$ & $7.558 \pm 0.065$ & $137.15 \pm 0.01$ & & V & $6.280 \pm 0.107$ & $137.78 \pm 0.03$ \\
\hline & $R$ & $7.172 \pm 0.095$ & $139.50 \pm 0.02$ & & $R$ & $5.922 \pm 0.047$ & $138.91 \pm 0.01$ \\
\hline & $I$ & $5.998 \pm 0.061$ & $138.00 \pm 0.01$ & & $I$ & $4.924 \pm 0.101$ & $139.14 \pm 0.03$ \\
\hline 30 & $U$ & $7.662 \pm 0.138$ & $136.02 \pm 0.02$ & 55 & $U$ & - & - \\
\hline & $B$ & $8.068 \pm 0.113$ & $137.75 \pm 0.02$ & & $B$ & $0.234 \pm 0.036$ & $143.80 \pm 0.20$ \\
\hline & $V$ & $8.837 \pm 0.081$ & $137.55 \pm 0.02$ & & V & $0.444 \pm 0.051$ & $148.42 \pm 0.17$ \\
\hline & $R$ & $8.259 \pm 0.057$ & $138.93 \pm 0.01$ & & $R$ & $0.512 \pm 0.093$ & $192.29 \pm 0.25$ \\
\hline & $I$ & $6.877 \pm 0.060$ & $137.45 \pm 0.01$ & & $I$ & $0.448 \pm 0.214$ & $130.61 \pm 0.75$ \\
\hline 35 & $U$ & $5.527 \pm 0.281$ & $144.16 \pm 0.06$ & 61 & $U$ & - & - \\
\hline & $B$ & $6.949 \pm 0.042$ & $138.91 \pm 0.01$ & & $B$ & $6.588 \pm 0.072$ & $136.77 \pm 0.02$ \\
\hline & $V$ & $6.885 \pm 0.036$ & $140.48 \pm 0.01$ & & V & $6.536 \pm 0.034$ & $137.81 \pm 0.08$ \\
\hline & $R$ & $6.363 \pm 0.024$ & $140.13 \pm 0.01$ & & $R$ & $6.195 \pm 0.038$ & $137.03 \pm 0.01$ \\
\hline & $I$ & $5.533 \pm 0.035$ & $139.80 \pm 0.01$ & & $I$ & $5.160 \pm 0.080$ & $137.99 \pm 0.02$ \\
\hline
\end{tabular}

Notes. The star number is its WEBDA designation and the degree of polarization $p(\%)$ and position angle $\theta$ measurements in the Johnson-Cousins $U B V R I$ filters are reported. 

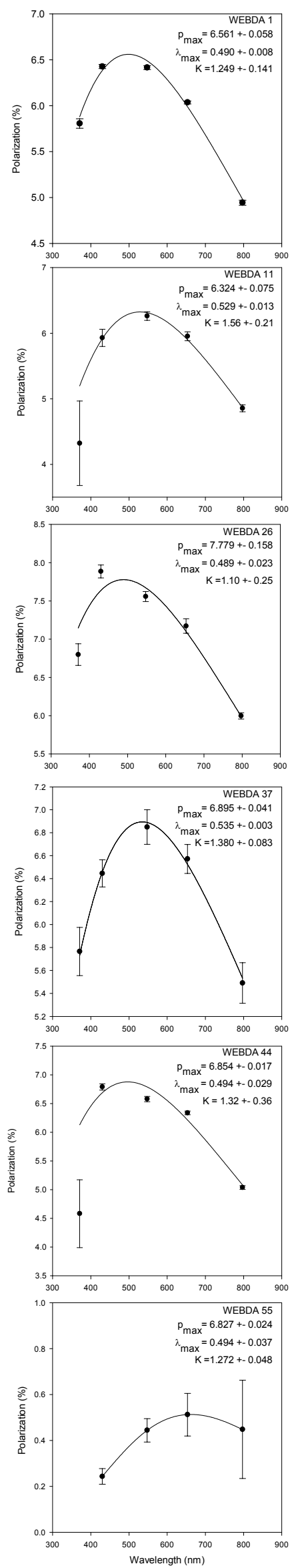
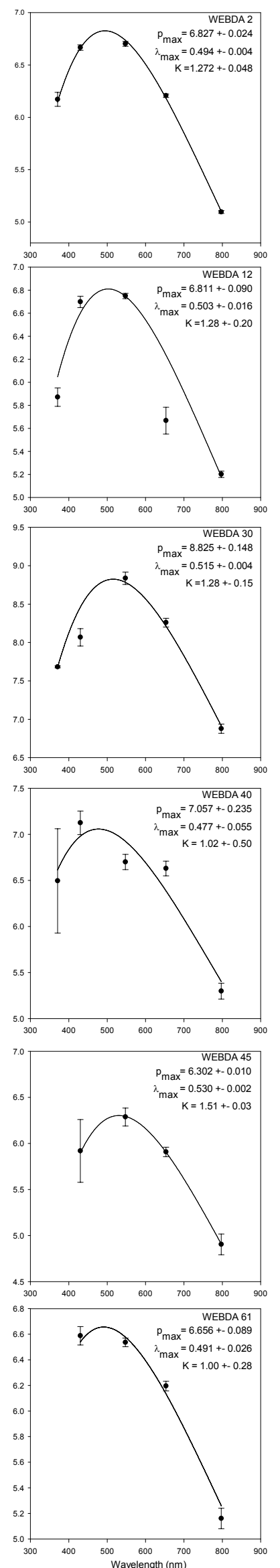
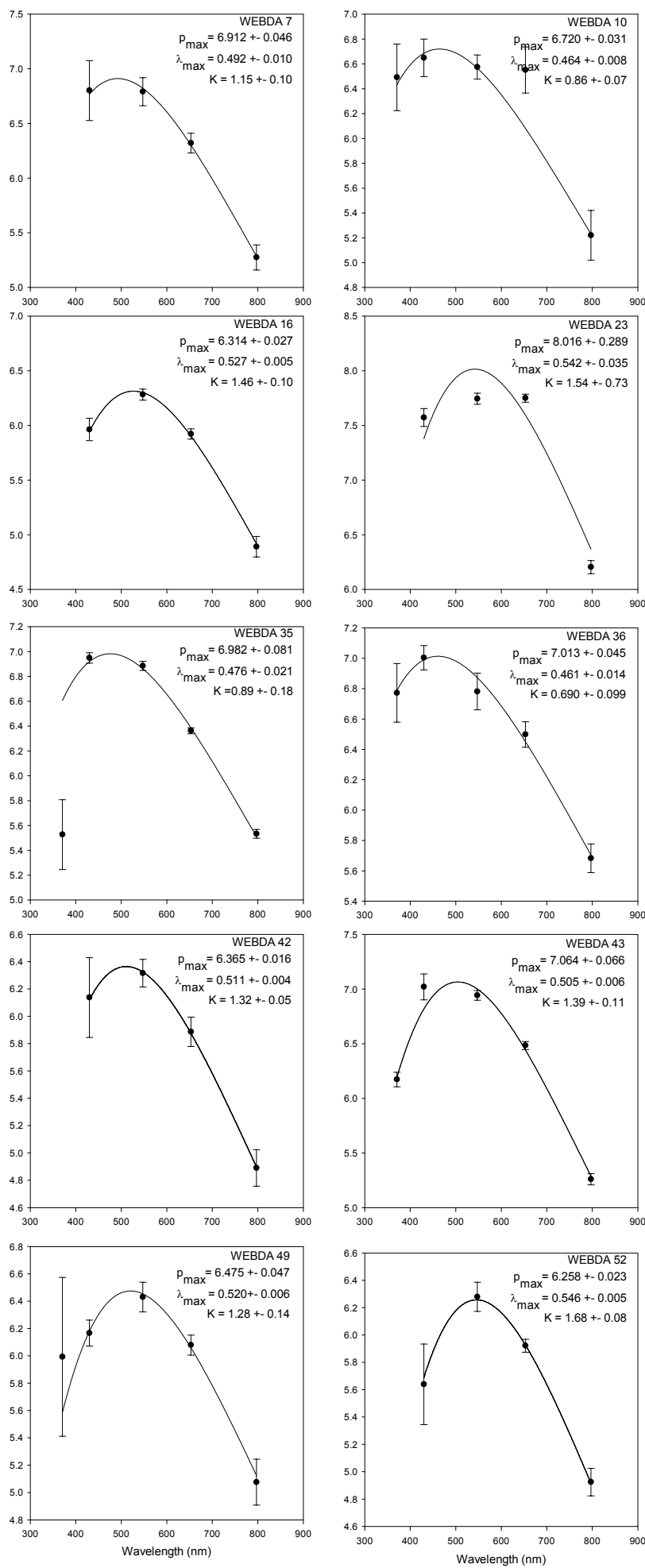

Fig. 1. Serkowski curves for the 22 stars studied here. 
and Weitenbeck et al. (2008, WHC, hereafter). WHC present measurements of the position angles and degree of maximum polarization of 7 cluster stars and 22 stars in the cluster vicinity. A comparison of their measurements with our measurements that are presented in Table 2 and shown in Fig. 3 indicates a good agreement.

The evaluation of the total-to-selective extinction toward NGC 1502 has been the subject of many investigations. A smaller value of $R$ at the Galactic longitude of NGC 1502 should be expected from the oscillatory nature of the maximum wavelength of stellar polarization, $\lambda_{\max }=0.545+0.03 \sin (l+$ $175^{\circ}$, Whittet 1977). At the longitude of NGC 1502 and given that $R=5.6 \lambda_{\max }$ a value of $R=2.94$ is likely. The study by Crézé (1972) on the variation of $R$ with Galactic longitude also indicates $R \lesssim 3$ in the longitude interval where NGC 1502 is located. Based on a small sample of cluster stars, WHC found an average $R$ of about 2.64. As they note, this is below the average Galactic value of 3.1, but consistent with the photometry-based result of $R=2.42 \pm 0.09$ by Tapia et al. (1991). An $R$ smaller than average, $2.57 \pm 0.27$, was also found by Pandey et al. (2003).

Our sample appears to be the largest one presently available of stars located within a small field of view centered on the cluster. From the polarization measurements in Table 2, the average value of $R$ is $2.83 \pm 0.03$, the lowest value being 2.58 and the highest 3.06. This value is higher than some of the previous estimates, but still lower than the Galactic average. We compared our measurements to those by WHC. For three of their distant field stars (HD 25090, HD 25443, and HD 24992), WHC found that $\lambda_{\max }$ ranged from $4930 \AA$ to $5050 \AA$, which when using $R=5.6 \lambda_{\max }$ gives a range of 2.76-2.83, consistent with our value of $R$ for the cluster itself. A weighted average of the values of $R$ listed in Table 3 by WHC yields $2.79 \pm 0.01$, while the three cluster stars included there give $2.70 \pm 0.01$. While observational evidence exists to support a value of $R$ lower than the Galactic average for this region, a very low value would be unlikely.

A stellar sample with homogeneous estimates of color excess and distance that are supplemented with optical polarimetry can be useful to locate the polarizing interstellar dust. Given the uniformity of the polarization across the field of NGC 1502 in both $p_{\max }$ and $\lambda_{\max }$, it appears that the dust responsible for polarization is in the foreground of the cluster, probably not more distant than $500 \mathrm{pc}$ and relatively uniformly distributed. This is discussed in connection to the photometric results in Sect. 4, but is also suggested in some of the previous investigations. For example, Tapia et al. (1991) found that the anomalous extinction yielding the small value of $R$ originated at an optical depth of $E(B-V) \geq 0.35$, which is relatively far in front of the cluster. This led to the conclusion that at larger distances along the line of sight (all the way to the cluster) the dust grain sizes are biased toward much smaller values compared to those in the diffuse ISM. We also note that two of the field stars listed by WHC, BD+61 677 and HD 25443, have similar values of $R$ (2.86 and 2.84) but quite different distances, $0.54 \pm 0.10$ and $2.5 \pm 1.9 \mathrm{kpc}$, respectively, as determined from their TGAS DR1 Gaia parallaxes. Since the Gaia parallaxes are not yet reliable for distances larger than $1 \mathrm{kpc}$, we looked into determining the distance by other means. Unfortunately, BD+61 677 has no $U B V$ photometry available. Using the Tycho-2 data $(B=10.04 \mathrm{mag}$, $V=9.6 \mathrm{mag}$ ) and the only available spectral type determination as A2 (Dombrovskii \& Hagen-Thorn 1964) yields a spectroscopic distance modulus of $6.9 \mathrm{mag}$, corresponding to a distance of $0.24 \mathrm{kpc}$. We note that the error in the spectroscopic distance

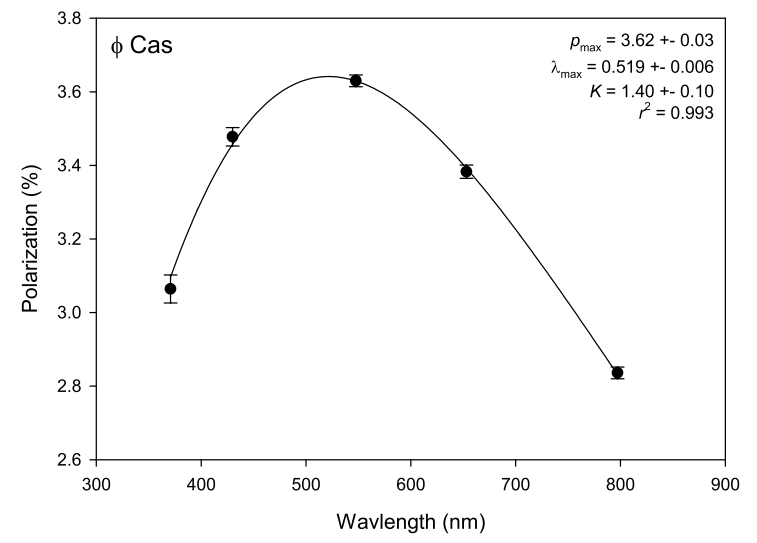

Fig. 2. Serkowski curve for the standard star HD 7927.

Table 2. Polarimetric data of stars toward NGC 1502.

\begin{tabular}{lccccccc}
\hline \hline Star & $p_{\max }(\%)$ & $\sigma_{p}$ & $\lambda_{\max }$ & $\sigma_{\lambda}$ & $K$ & $\sigma_{K}$ & $r^{2}$ \\
\hline 1 & 6.56 & 0.06 & 0.499 & 0.008 & 1.25 & 0.14 & 0.988 \\
2 & 6.83 & 0.02 & 0.494 & 0.004 & 1.27 & 0.05 & 0.999 \\
7 & 6.91 & 0.05 & 0.492 & 0.010 & 1.15 & 0.10 & 0.999 \\
10 & 6.72 & 0.03 & 0.464 & 0.008 & 0.86 & 0.07 & 0.997 \\
11 & 6.32 & 0.07 & 0.529 & 0.013 & 1.56 & 0.21 & 0.989 \\
12 & 6.81 & 0.09 & 0.503 & 0.016 & 1.28 & 0.20 & 0.983 \\
16 & 6.31 & 0.03 & 0.527 & 0.005 & 1.46 & 0.10 & 0.998 \\
23 & 8.02 & 0.29 & 0.542 & 0.035 & 1.54 & 0.73 & 0.889 \\
26 & 7.79 & 0.16 & 0.489 & 0.023 & 1.10 & 0.25 & 0.973 \\
30 & 8.83 & 0.15 & 0.515 & 0.004 & 1.28 & 0.15 & 0.972 \\
35 & 6.98 & 0.08 & 0.476 & 0.021 & 0.89 & 0.18 & 0.982 \\
36 & 7.01 & 0.04 & 0.461 & 0.014 & 0.69 & 0.10 & 0.992 \\
37 & 6.90 & 0.04 & 0.535 & 0.003 & 1.38 & 0.08 & 0.993 \\
40 & 7.06 & 0.24 & 0.477 & 0.055 & 1.02 & 0.50 & 0.911 \\
42 & 6.36 & 0.02 & 0.511 & 0.004 & 1.32 & 0.05 & 0.999 \\
43 & 7.06 & 0.07 & 0.505 & 0.006 & 1.39 & 0.11 & 0.991 \\
44 & 6.85 & 0.02 & 0.494 & 0.029 & 1.32 & 0.36 & 0.966 \\
45 & 6.30 & 0.01 & 0.531 & 0.002 & 1.51 & 0.03 & 0.999 \\
49 & 6.48 & 0.05 & 0.520 & 0.006 & 1.28 & 0.14 & 0.985 \\
52 & 6.26 & 0.02 & 0.546 & 0.005 & 1.68 & 0.08 & 0.999 \\
55 & 0.57 & 0.02 & 0.614 & 0.014 & 6.95 & 0.94 & 0.995 \\
61 & 6.66 & 0.09 & 0.491 & 0.026 & 1.00 & 0.28 & 0.978 \\
\hline
\end{tabular}

Notes. The maximum degree of polarization $p_{\max }$, wavelength of maximum polarization $\lambda_{\max }$, and the parameter $K$, along with their uncertainties and goodness of fit are shown.

cannot be reliably estimated in this case because of the unknown uncertainties in the spectral classification and the photometry. HD 25443 (HIP 19139) is classified as B0.5 III by several authors and more recently by Walker (1963). The available $U B V$ data (the most recent from Kitamura \& Yamasaki 1972) yield a spectroscopic distance modulus of $9.76 \mathrm{mag}(0.9 \mathrm{kpc})$. The HIPPARCOS parallax of this star $(1.45 \pm 0.77$ mas $)$ results in a distance of $0.69_{-0.24}^{+0.7} \mathrm{kpc}$. Thus, all currently possible estimates imply that these two field stars are indeed spread over distance, suggesting that the polarizing dust should be closer than about $500 \mathrm{pc}$. For these photometry-based estimates we used the value obtained here, $R=2.83$.

The interstellar polarization is predominantly caused by large grains (grain size $a>0.1 \mu \mathrm{m}$ ), however, the size distribution in the ISM is weighted more heavily toward smaller grain size (Mathis et al. 1977; Clayton et al. 2003). Therefore, not all 


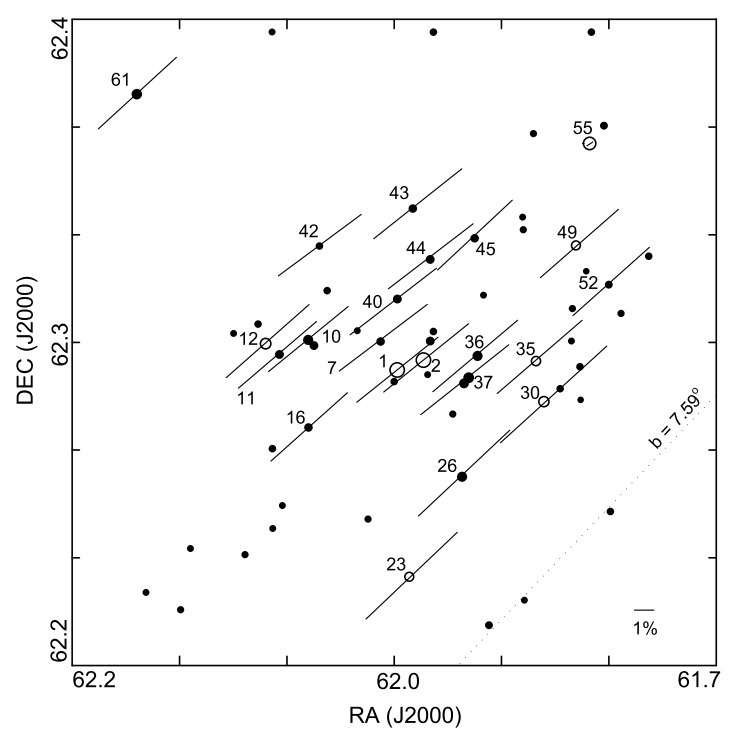

Fig. 3. Polarization vectors map. Open symbols are used for the stars included in the first Gaia data release.

dust grains contributing to extinction contribute to polarization (Whittet 2004); smaller grains continue contributing to extinction at shorter wavelengths whereas polarization declines. In particular, the distinct $\lambda \approx 0.22 \mu \mathrm{m}$-rise in extinction is almost always absent from polarization curves, suggesting that these small carbon grains do not contribute significantly to polarization, possibly because they are not aligned like the larger grains. If the grain distribution found by Tapia et al. (1991) is weighted towards smaller grain sizes that most likely arises in the vicinity of NGC 1502, then these smaller grains are not likely to be a source of significant polarization. This leaves the larger grain sizes to produce the observed polarization. For long dielectric cylinders of radius $a$ and refractive index $n$, the grain size is related to the wavelength of maximum polarization by $\lambda_{\max } \approx 2 \pi a(n-1)$ (Whittet 2003). For silicate grains $(n=1.6)$, the average $\lambda_{\max } \sim 0.5 \mu \mathrm{m}$ found here yields a grain size of the order of $0.13 \mu \mathrm{m}$.

Given the uniformity of both $p_{\max }$ and $\lambda_{\max }$ in and around the cluster, as well as the grain sizes implied by the polarization measurements, it seems likely that the polarization in this region arises from aligned dust grains in the ISM that are not associated with the dust in the vicinity of NGC 1502.

\section{Membership probabilities}

The analysis of the membership probabilities for stars within cluster areas is not straightforward. However, this analysis is vital for any further estimation of the cluster parameters. Netopil et al. (2015) published a critical assessment of the available cluster parameters. They found that the largest compilations probably include at least $20 \%$ problematic objects, for which the parameters differ significantly.

For our study of NGC 1502, we investigated the individual membership probabilities of our targets published in the literature. We compared the results from Kharchenko et al. (2013) based on the proper motions of the PPMXL (Roeser et al. 2010) and color-magnitude diagrams of the 2MASS catalog (Skrutskie et al. 2006) with the ones from Dias et al. (2014) who used the UCAC4 (Zacharias et al. 2013). Both sources were searched for common entries of our targets. Table 3 lists the probabilities of both references. This table includes all stars with $U B V$ and
Table 3. Membership probabilities (\%) taken from Kharchenko et al. (2013; Pkin, PJKs, and PJH), Dias et al. (2014; D14) and our estimates (HSOY, PPMXL, and UCAC5).

\begin{tabular}{|c|c|c|c|c|c|c|c|}
\hline Star & Pkin & PJKs & PJH & PD14 & HSOY & PPMXL & UCAC5 \\
\hline 1 & 63 & 100 & 83 & 99 & 0 & 66 & 54 \\
\hline 2 & 18 & 73 & 70 & 0 & 0 & 23 & 0 \\
\hline 7 & 99 & 100 & 100 & 99 & 97 & 98 & 67 \\
\hline 9 & & & & 98 & 30 & 46 & \\
\hline 10 & 43 & 91 & 100 & 98 & 30 & 46 & 39 \\
\hline 11 & 97 & 0 & 9 & 99 & 1 & 97 & 96 \\
\hline 12 & 56 & 70 & 96 & 99 & 4 & 83 & 38 \\
\hline 13 & 0 & 51 & 72 & 78 & 0 & 0 & 29 \\
\hline 14 & 90 & 0 & 6 & 98 & 55 & 91 & 61 \\
\hline 15 & 62 & 0 & 0 & 57 & 25 & 67 & 4 \\
\hline 16 & 74 & 100 & 100 & 99 & 46 & 73 & 68 \\
\hline 17 & 98 & 74 & 61 & 98 & 94 & 98 & 86 \\
\hline 18 & 98 & 99 & 100 & 98 & 90 & 98 & 88 \\
\hline 23 & 100 & 34 & 99 & 99 & 0 & 100 & 92 \\
\hline 24 & 51 & 100 & 100 & & 67 & 53 & 77 \\
\hline 26 & 79 & 93 & 64 & 99 & 60 & 82 & 66 \\
\hline 27 & 24 & 85 & 100 & 99 & 53 & 28 & 79 \\
\hline 29 & 33 & 0 & 0 & 0 & 2 & 38 & 0 \\
\hline 30 & 46 & 100 & 96 & 99 & 34 & 50 & 43 \\
\hline 34 & 20 & 100 & 100 & 96 & 0 & 24 & 85 \\
\hline 35 & 66 & 100 & 100 & 99 & 74 & 68 & 38 \\
\hline 36 & 95 & 91 & 64 & 99 & 89 & 96 & 32 \\
\hline 37 & & & & 84 & 61 & 52 & 99 \\
\hline 38 & & & & 84 & 0 & 0 & 35 \\
\hline 40 & 44 & 92 & 100 & 99 & 20 & 47 & 86 \\
\hline 41 & 94 & 100 & 100 & 97 & 83 & 94 & 79 \\
\hline 42 & 73 & 100 & 100 & 98 & & 76 & 85 \\
\hline 43 & 70 & 93 & 99 & 99 & 98 & 72 & 93 \\
\hline 44 & 12 & 92 & 100 & 99 & 17 & 14 & 83 \\
\hline 45 & 58 & 99 & 85 & 99 & 89 & 72 & 93 \\
\hline 49 & 75 & 82 & 81 & 99 & 0 & 47 & 86 \\
\hline 51 & 77 & 100 & 100 & 87 & 11 & 80 & 33 \\
\hline 52 & 74 & 57 & 72 & 98 & 97 & 75 & 3 \\
\hline 55 & 0 & 26 & 23 & 0 & 0 & 0 & 0 \\
\hline 59 & 22 & 0 & 0 & & 0 & 25 & 0 \\
\hline 60 & 42 & 100 & 100 & & 16 & 45 & 0 \\
\hline 61 & & & & & 1 & 77 & 62 \\
\hline 78 & 31 & 55 & 96 & & 84 & 33 & 81 \\
\hline 79 & 97 & 100 & 100 & 96 & 96 & 97 & 63 \\
\hline
\end{tabular}

uvby $\beta$ data in the field of the cluster. The stars are identified by their WEBDA numbers.

First of all, we compared the membership probabilities (Kharchenko et al. 2013) derived from the PPMXL (Pkin) and the color-magnitude diagrams (PJKs and PJH). This comparison is shown in Fig. 4, upper panel. There is absolutely no correlation. This means that stars that are lying on the fitted isochrone using the cluster parameters are kinematically non-members and implies the need to also include kinematic data for the membership probability estimation. Even more surprising is the comparison with the results from Dias et al. (2014) (middle panel). The latter lists almost all stars as true members of NGC 1502 with probabilities of $98 \%$ or $99 \%$ (Table 3). This result is highly unlikely also in the light of our analysis using the UCAC5 catalog (Zacharias et al. 2017) and the Hot Stuff for One Year (HSOY; Altmann et al. 2017) catalog. The HSOY is the successor of the PPMXL and the Gaia DR1. 


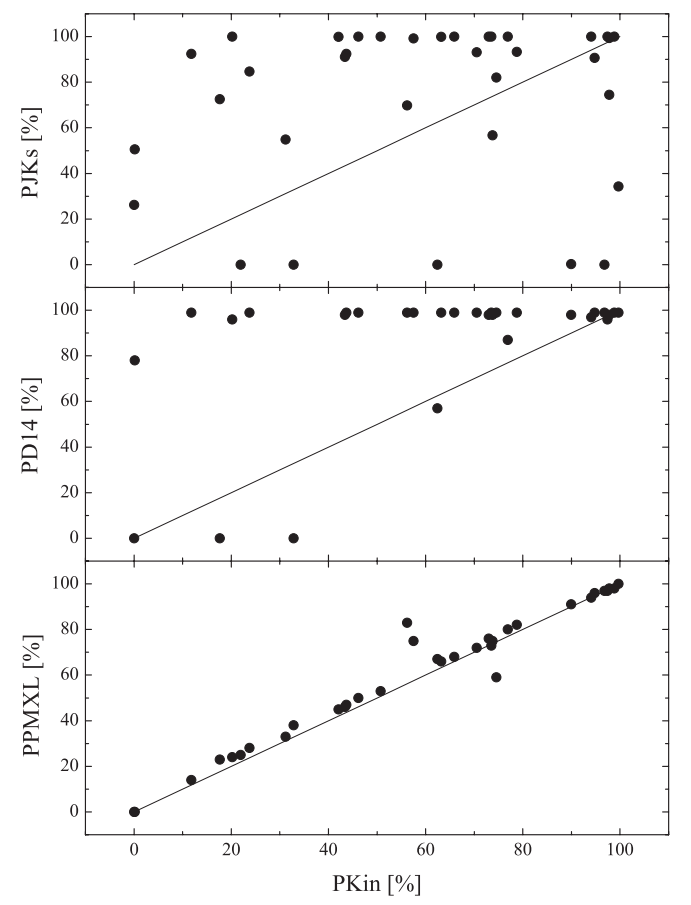

Fig. 4. Membership probabilities from Kharchenko et al. (2013) derived from the PPMXL (Pkin) and the color-magnitude diagram (PJKs); (Dias et al. 2014, PD14) and our result for the PPMXL (Table 3).

Subsequently, we employed the method given by Balaguer-Núnez et al. (1998) to estimate the kinematic membership probabilities of our targets. This method takes both the errors of the mean cluster and the stellar proper motions into account. The basic idea of the nonparametric method for the cluster-field separation in the two-dimensional proper motion space is the empirical determination of the cluster and field stars' distributions without any assumption about their shape. We used the kernel estimation technique (with a circular Gaussian kernel function) to derive the data distributions. The proper motions of the stars were taken from the PPMXL, HSOY and UCAC5 catalog and the corresponding membership probabilities calculated here are listed in the last three columns of Table 3. Additionally, we compared the results of this method with those of the algorithm published by Javakhishvili et al. (2006), yielding excellent agreement. To calculate the mean cluster proper motion, the values listed in Zejda et al. (2012), Kharchenko et al. (2013) and Dias et al. (2014) were taken (five in total). The listed errors were used as reciprocal weights resulting in a cluster proper motion of $\mu_{\alpha} \cos \delta=+0.07(25)$ and $\mu_{\delta}=-0.63(49)$ mas $\mathrm{yr}^{-1}$, respectively.

Figure 4 (lower panel) shows the comparison of our results based on the PPMXL with that of Kharchenko et al. (2013). There is an excellent agreement between these two parameters on the basis of the same source but on widely different methods. This promotes further confidence in our analysis.

We compared the proper motions of our targets taken from the HSOY and UCAC5 (Fig. 5, upper and middle panel). Besides a relatively large scatter, the $\mu_{\alpha} \cos \delta$ values show no obvious systematic errors with just two outliers. For $\mu_{\delta}$ there is a zero offset and a possible systematic relation for positive values visible. Although these effects seem relatively small, they have a significant influence on the membership probabilities (lower panel of Fig. 5). We also notice that about ten stars are, according to the HSOY, non-members with a probability of zero percent. This is caused by the very small errors $\left(<1 \mathrm{mas} \mathrm{yr}^{-1}\right)$ listed in this catalog. Those errors are derived based on a global
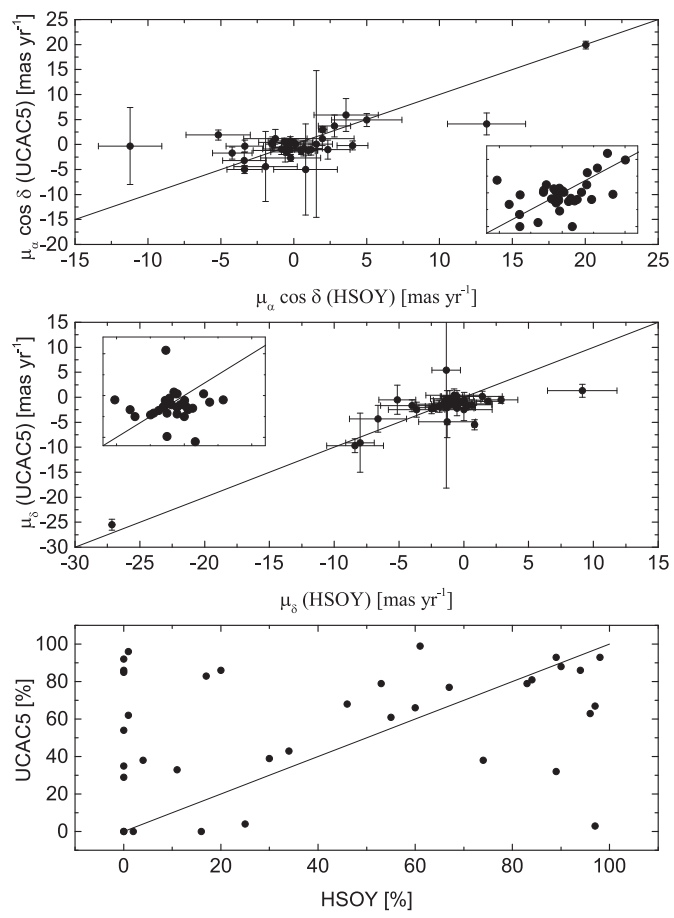

Fig. 5. A comparison of the proper motions taken from the HSOY and UCAC5 (upper and middel panels) as well as the membership probabilities derived from them (lower panel).

statistical assessment of the whole data set. Whether or not these are realistic is a question that must be addressed in the future when the complete data from the Gaia satellite mission has been published. The source of the individual stellar proper motion is therefore still most important for star clusters such as NGC 1502 which is only about $1 \mathrm{kpc}$ from the Sun. The first major improvement of this situation will be the publication of proper motions only depending on the measurements from the Gaia satellite mission.

\section{Photometric analysis}

In this section we consider available $u v b y \beta$ and $U B V$ photometric data for the $\mathrm{O}$ and B-type stars in the field of NGC 1502, as well as their MK classification to infer the basic parameters of the cluster. The $u v b y \beta$ system is an intermediate-band system and is considered to be useful for yielding physical stellar parameters. The detailed classical description of the $u v b y \beta$ photometric quantities and their application can be found in Strömgren (1966). In the O-B9 spectral range considered here, the $\beta$ index is a measure of the strength of the $\mathrm{H} \beta$ line and is thus a luminosity indicator that is essential for the calculation of the absolute magnitude $M_{V}$. The $(b-y)$ index is a temperature indicator, providing the true stellar color, and therefore the interstellar reddening. The color difference $c_{1}=(u-v)-(v-b)$ is a measure of the Balmer jump and an indicator of the effective temperature for O-B9 stars that is also used in the $M_{V}$ calculations. In general, multi-color stellar photometry of high accuracy and precision, especially in the optical spectral range, is very useful in the study of star-forming fields and young open clusters of relatively high and often variable interstellar extinction. In many of these cases usually only small samples of $\mathrm{O}$ - and B-type stars are available to infer interstellar reddening and stellar distances and to construct the HR diagram yielding the cluster's or association's age. Since in the case of NGC 1502 
Table 4. Data sources of $U B V$ electrophotometry of stars in NGC 1502.

\begin{tabular}{lcc}
\hline \hline Reference & $\begin{array}{c}\text { WEBDA } \\
\text { source }\end{array}$ & $\begin{array}{c}\text { Number } \\
\text { of stars }\end{array}$ \\
\hline Lutz \& Lutz (1977) & 285 & 3 \\
Purgathofer (1964) & 821 & 13 \\
Reimann \& Pfau (1987) & 907 & 22 \\
Guetter (1964) & 1321 & 2 \\
Hoag et al. (1961) & 1341 & 26 \\
Eggen (1968) & 1385 & 2 \\
Kitamura \& Yamasaki (1972) & 1432 & 2 \\
\hline
\end{tabular}

Notes. The WEBDA identification number for the sources and the number of stars per source are given in Cols. 2 and 3.

a precise differential reddening evaluation is needed, we first address the analysis based on the $u v b y \beta$ photometry.

\subsection{Evaluation of existing $U B V$ and $u v b y \beta$ datasets for NGC 1502}

With the accumulation of larger amounts of photometric data inconsistencies between different datasets need to be evaluated. This has previously been discussed by Crawford (1994a, 1999) stressing discrepancies in the $U B V$ and $u v b y \beta$ photometries of several young Galactic clusters. Here we reiterate the issue in the case of NGC 1502 .

Photoelectric $U B V$ photometry of stars in NGC 1502 has been published by several authors, as listed in Table 4 . In this table, the reference source is given in the first column, followed by the WEBDA reference source identification number and the number of stars per reference source. The WEBDA reference source identifications will be used hereafter when referring to the original datasets. In addition, the WEBDA numbering system is used for the star identification throughout the paper.

We analyzed the distribution of the residuals in $V, U-B$, and $B-V$ for sources 821 and 907 in comparison to source 1341, which contains the largest sample, and found no systematic trends present. Thus we calculated the average $V, U-B$, and $B-V$ values of the available photoelectric photometries and then used them to evaluate the existing sources of uvby photometry. Table 5 lists these average values based on all available sources of photoelectric $U B V$ photometry. Some of these sets consist of only 1 or 2 stars in common with the rest of the sources, but we included them anyway. The standard deviation of the mean, denoted $\sigma$ hereafter, is indicated where available.

At present, there are several sets of original and homogenized $u v b y \beta$ data available for the field of NGC 1502. Existing original photoelectric and CCD $u v b y \beta$ datasets are listed in Table 6 and consist of overlapping samples for most of the bright early-type stars. This table is constructed in a similar way as Table 4. The last source included (Handler 2011, denoted H2011 hereafter) is relatively recent and does not appear in the WEBDA database.

The cluster is included in the $u v b y \beta$ homogenized catalog by Hauck \& Mermilliod (1998), which is based on data published until the end of 1998, and also in the recent catalog by Paunzen (2015). The former compilation uses procedures that give a lower weight to discrepant values, while the latter provides averaged (without weighting) photometric values together with the standard deviation of the mean, where available. In addition, homogenized photometry of 21 stars in the field of the cluster is presented by Crawford (1994b, his Table 3), combining (after a number of zero-point corrections) his observations of 14 stars (WEBDA source 515) with previously obtained photometries.
Table 5. Averaged $U B V$ photometry based on sources listed in Table 4 .

\begin{tabular}{|c|c|c|c|c|c|c|c|}
\hline Star & $V$ & $\sigma_{V}$ & $B-V$ & $\sigma_{B-V}$ & $U-B$ & $\sigma_{U-B}$ & MK \\
\hline 1 & 6.96 & 0.02 & 0.47 & 0.03 & -0.52 & 0.01 & B0III \\
\hline 2 & 6.99 & 0.04 & 0.42 & 0.02 & -0.54 & 0.02 & $\mathrm{O} 9 \mathrm{IV}+\mathrm{B} 0.5 \mathrm{~V}$ \\
\hline 7 & 11.53 & 0.01 & 0.59 & 0.04 & +0.02 & 0.07 & B8 \\
\hline 10 & 9.82 & & 0.57 & & -0.22 & & B3 \\
\hline 11 & 10.88 & & 0.63 & & -0.03 & & $\mathrm{~B} 1.5 \mathrm{~V}$ \\
\hline 12 & 9.42 & 0.01 & 0.47 & 0.02 & -0.41 & 0.03 & B2 \\
\hline 13 & 12.76 & & 0.56 & & +0.10 & & \\
\hline 14 & 13.67 & & 0.99 & & +0.69 & & \\
\hline 15 & 12.73 & & 1.59 & & +1.35 & & \\
\hline 16 & 11.61 & 0.03 & 0.61 & 0.02 & -0.01 & 0.01 & B5V \\
\hline 17 & 13.56 & & 0.74 & & +0.39 & & \\
\hline 18 & 13.84 & & 0.81 & & +0.54 & & \\
\hline 23 & 10.74 & 0.02 & 0.62 & 0.00 & -0.11 & 0.00 & B3V \\
\hline 24 & 12.76 & & 0.72 & & +0.23 & & \\
\hline 26 & 9.64 & 0.04 & 0.56 & 0.02 & -0.34 & 0.02 & $\mathrm{~B} 1.5 \mathrm{~V}$ \\
\hline 27 & 12.44 & & 0.55 & & -0.08 & & \\
\hline 29 & 12.93 & & 0.93 & & +0.69 & & \\
\hline 30 & 9.67 & 0.01 & 0.58 & 0.01 & -0.37 & 0.02 & $\mathrm{~B} 1 \mathrm{~V}$ \\
\hline 34 & 14.24 & & 1.27 & & +0.42 & & \\
\hline 35 & 10.50 & 0.01 & 0.51 & 0.02 & -0.29 & 0.01 & B3V \\
\hline 36 & 9.79 & 0.04 & 0.56 & 0.03 & -0.32 & 0.01 & B8V \\
\hline 37 & 9.30 & & 0.49 & & -0.28 & & $\mathrm{~B} 1.5 \mathrm{~V}$ \\
\hline 40 & 11.28 & 0.11 & 0.51 & 0.03 & -0.18 & 0.01 & B2V \\
\hline 41 & 12.93 & & 0.84 & & +0.52 & & A2 \\
\hline 42 & 12.57 & 0.06 & 0.64 & 0.05 & +0.24 & 0.02 & B9 \\
\hline 43 & 11.37 & 0.01 & 0.56 & 0.02 & -0.09 & 0.03 & \\
\hline 44 & 10.80 & 0.01 & 0.60 & 0.00 & -0.20 & 0.06 & B2V \\
\hline 45 & 11.43 & 0.03 & 0.62 & 0.02 & -0.05 & 0.01 & $\mathrm{~B} 4.5 \mathrm{~V}$ \\
\hline 49 & 10.73 & 0.01 & 0.53 & 0.04 & -0.24 & 0.01 & B3V \\
\hline 51 & 13.17 & & 0.92 & & +0.28 & & \\
\hline 52 & 12.25 & & 0.70 & & +0.45 & & B9 \\
\hline 55 & 7.94 & 0.02 & 0.06 & 0.02 & +0.03 & 0.02 & A2V:n \\
\hline 59 & 7.96 & 0.05 & 1.20 & 0.01 & +0.78 & 0.01 & G0III \\
\hline 60 & 9.64 & 0.04 & 0.32 & 0.00 & +0.14 & 0.01 & A3V \\
\hline 61 & 9.58 & 0.02 & 0.44 & 0.01 & -0.35 & 0.02 & B2V \\
\hline 76 & 10.39 & & 0.58 & & -0.26 & & B8 \\
\hline 78 & 10.92 & 0.01 & 0.50 & 0.00 & -0.19 & 0.01 & B5V \\
\hline 79 & 14.69 & & 1.28 & & +0.31 & & \\
\hline
\end{tabular}

Notes. The available MK classification is listed in the last column.

Table 6. Data sources of $u v b y \beta$ electrophotometry or CCD photometry of stars in NGC 1502.

\begin{tabular}{lcc}
\hline \hline Reference & $\begin{array}{c}\text { WEBDA } \\
\text { source }\end{array}$ & $\begin{array}{c}\text { Number } \\
\text { of stars }\end{array}$ \\
\hline Crawford et al. (1971) & 44 & 2 \\
Shaw (1975) & 117 & 1 \\
Schmidt (1984) & 361 & 1 \\
Reimann \& Pfau (1987) & 403 & 22 \\
Tapia et al. (1991) & 467 & 21 \\
Delgado et al. (1992) & 471 & 22 \\
Crawford (1994b) & 515 & 14 \\
Handler (2011) & - & 11 \\
\hline
\end{tabular}

Notes. The WEBDA identification number for the sources and the number of stars per source are given in Cols. 2 and 3. 


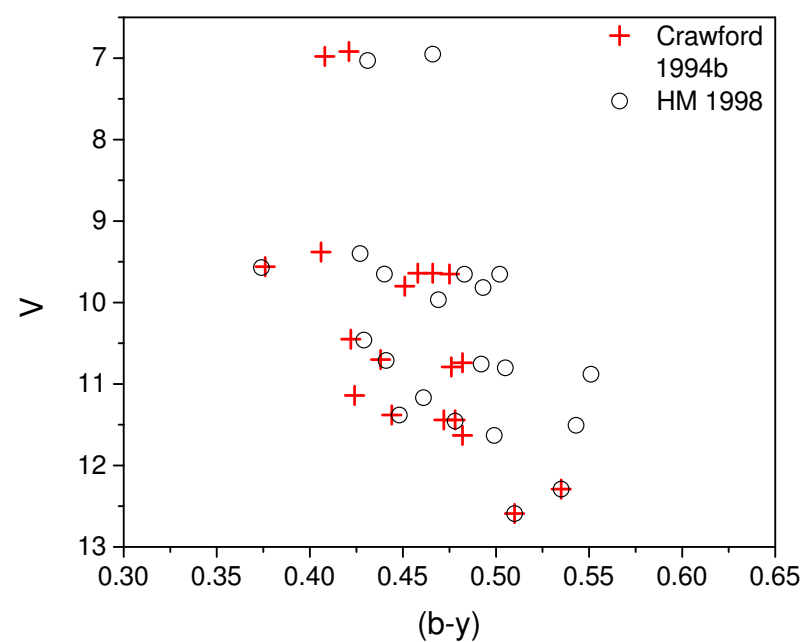

Fig. 6. The $V$ vs. $b-y$ diagram based on Crawford (1994b) and the catalog of Hauck \& Mermilliod (1998).

Figure 6 presents the $V$ vs. $b-y$ diagram showing the homogenizations by Crawford (1994b) and Hauck \& Mermilliod (1998). The $b-y$ indices based on Crawford (1994b) appear less reddened than the data from the Hauck \& Mermilliod (1998) catalog. This is due to the more rigorous procedure applied in Crawford (1994b) when dealing with reddened OB stars.

In any case, such discrepancies, although small, may be sufficient to affect the color excess determinations and may yield controversial conclusions regarding possible differential reddening and the overall characteristics of the cluster. Since we are aiming at precise extinction determinations for the individual stars, the consistency of the existing $u v b y \beta$ photometry should be additionally addressed using another approach, more specifically a comparison of the color excesses derived from $U B V$ and $u v b y$ data. For O- and B-type stars the color excess $E(b-y)$ is calculated via $b-y$ and $c_{1}$. Thus, direct comparisons between $E(b-y)$ and $E(B-V)$ should be helpful in detecting possible systematic deviations in $b-y$ and $c_{1}$. This will allow us to exclude deviating uvby datasets when constructing the average quantities. We note that for early-type stars this is a more reliable approach when evaluating systematic discrepancies in comparison to an evaluation of the $\left[c_{1}\right]$ vs. $\left[m_{1}\right]$ and $u-b$ vs. $U-B$ diagrams. In the O-B9 spectral range, $m_{1}$ is not used in the calculation of the color excesses or the absolute magnitudes. It is, however, sometimes more difficult to be precisely transformed to the standard system. Since it is included in the calculation of both $\left[m_{1}\right]$ and $u-b$, systematic errors in $m_{1}$ would certainly affect the appearance of the corresponding diagrams. This can lead to unnecessary exclusion of datasets otherwise providing reliable color excesses and absolute magnitudes. We also note that the $u-b$ quantity, which is often used to evaluate systematic error in the uvby photometry, is calculated as $u-b=2(b-y)+2 m_{1}+c_{1}$, accumulating in this way all possible (sometime systematic) errors in these three quantities.

This situation is illustrated in Figs. 7 and 8. Figure 7, top panel, presents the $\left[c_{1}\right]$ vs. $\left[m_{1}\right]$ diagram for the stars included in the individual sources listed in Table 6 (identified with their WEBDA source numbers). These datasets contain only a few later spectral types and we restricted the study to stars earlier than A0 type only. This figure also shows the $b-y$ vs. $B-V$ and $u-b$ vs. $U-B$ comparisons. Here we have used the average $U-B$ and $B-V$ values (Table 5). All three plots indicate systematic inconsistencies to some degree, depending
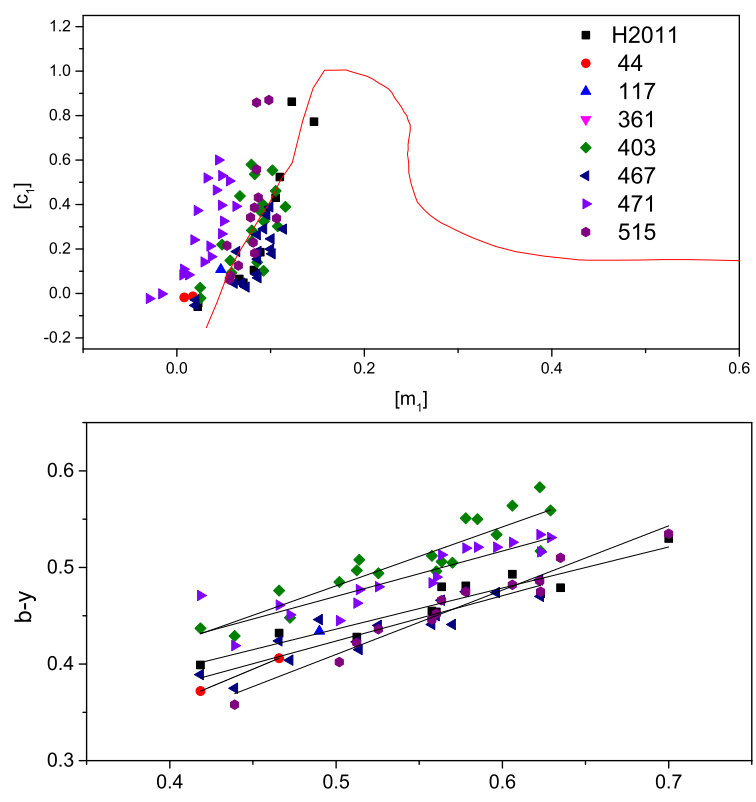

(B-V) average

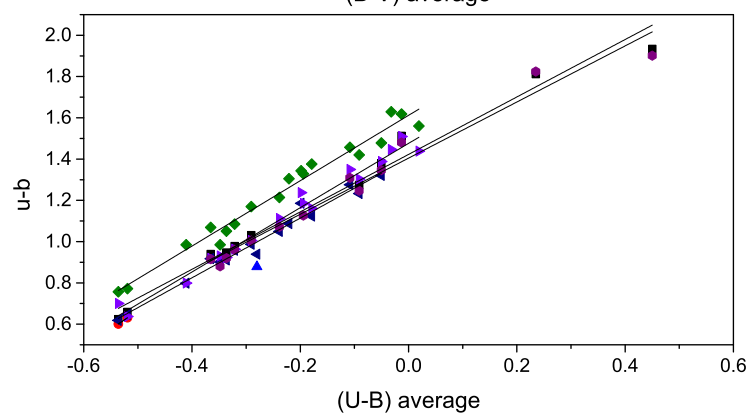

Fig. 7. Top: classification $\left[c_{1}\right]$ vs. $\left[m_{1}\right]$ diagram for the stars earlier than A0 spectral type with available $u v b y \beta$ photometry. Different datasets are marked with different symbols, as indicated on the panel, using the WEBDA source identifications listed in Table 6. The solid line on this diagram represents the main sequence (Crawford 1975, 1978). Middle: comparisons of the average $B-V$ to $b-y$ from the individual sources. Bottom: similar comparisons for the $U-B$ and $u-b$ quantities. Linear fits corresponding to the different datasets are shown on the last two panels.

on the considered quantities, which we reassess with the new homogenization.

Next we obtained the dereddened $U B V$ quantities corresponding to the averaged $U B V$ photometry using Eqs. (3)-(5; see also Crawford 1994b). The color excesses $E(b-y)$ and the dereddened $(b-y)_{0}$ and $c_{0}$ quantities are calculated via Eqs. (6)-(8), which are based on the calibration by Crawford (1978). Then we compared $E(b-y)$ and $E(B-V)$ for the sources listed in Table 6, using the accepted conversion $E(B-V)=E(b-y) / 0.74$ (see e.g., Straižys 1992). We found good agreement between the color excesses for all sources, except for 403, showing somehow larger deviation. As expected, sources showing offset on the $\left[c_{1}\right]$ versus $\left[m_{1}\right]$ diagram yielded color excesses $E(b-y)$ in agreement with the $E(B-V)$ values.

$$
\begin{aligned}
& (U-B)_{0}=1.242(U-B)-0.894(B-V), \\
& E(U-B)=(U-B)-(U-B)_{0} \\
& E(B-V)=E(U-B) / 0.72 \\
& (b-y)_{0}=\left(c_{1}-0.2(b-y)-1.14\right) / 9.79 \\
& E(b-y)=(b-y)-(b-y)_{0} \\
& c_{0}=c_{1}-0.2 E(b-y)
\end{aligned}
$$




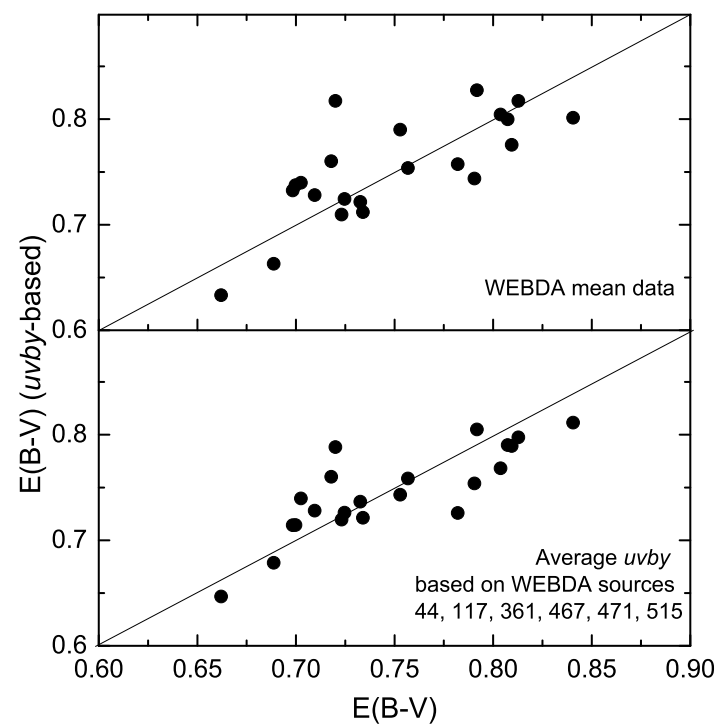

Fig. 8. Color excess $E(B-V)$ based of $u v b y$ photometry $(E(b-y) / 0.74)$ vs. $E(B-V)$ for the WEBDA mean data (top) and for the new homogenization based on our selection of sources (bottom).

Further we produced a combined uvby dataset averaging the quantities from all sources excluding only source 403. Figure 8 presents the comparisons of the $u v b y$-based color excess obtained via the WEBDA mean data (top) and the new averaged data (bottom). The WEBDA mean data refers to the homogenization accepted in the WEBDA database (and in the Hauck \& Mermilliod 1998 catalog). The statistical analysis we performed suggests that the new average performs slightly better in this comparison, showing a residual distribution closer to normal. This new homogenization is presented in Table 7 and adopted for the following analysis. This alternative approach is however possible only for clusters for which multiple photometric data sources are available and is not feasible when constructing large databases.

We noticed possible problems with W10, which could be misidentified in source 467. For W38 we adopt $V=9.825$, but the star may be variable in the range 9.25-10.4 in $V$. Only six of the sources listed in Table 6 provide $\mathrm{H} \beta$ measurements and the average is included in Table 7. For the adopted $\beta$ value of W55 we use only source 471 . We also note that the $u v b y \beta$ data for stars W5, as listed in WEBDA, might be actually for W6, and we omit both W5 and W6 from Table 7.

\section{2. $u v b y \beta$-based cluster parameters}

All stars with polarimetric measurements (Tables 1 and 2) have complete $u v b y \beta$ photometry in all homogenized sets. The $\left[c_{1}\right] /\left[m_{1}\right]$ diagram and also the $\beta /\left[c_{1}\right]$ diagram (not shown) clearly indicate them as B-type, except W55, which is an early A-type foreground star at about $190 \mathrm{pc}$ (this star is not included in the $\left[c_{1}\right] /\left[m_{1}\right]$ plot on Fig. 7.) The $\beta /\left[c_{1}\right]$ diagram is also useful in identifying emission-line objects, as they are located on average above the sequence of the normal B-type stars due to the effect of emission in the $\beta$ index. The majority of the B-type stars in our sample appear as main-sequence nonemission objects, except W42 and W52, which seem to be of later spectral types and slightly evolved. They are both classified as B9, which is consistent with their location on both diagrams.

Since there is no indication that the stars we consider are more luminous than luminosity class III, the calibration by Crawford (1978) is applied to calculate the color excess $E(b-y)$
Table 7. New homogenization of $u v b y \beta$ photometry.

\begin{tabular}{rccccc}
\hline \hline Star & $V$ & $b-y$ & $m_{1}$ & $c_{1}$ & $\beta$ \\
\hline 1 & 6.925 & 0.430 & -0.138 & 0.054 & 2.578 \\
2 & 6.990 & 0.411 & -0.124 & 0.067 & 2.591 \\
7 & 11.460 & 0.521 & -0.119 & 0.634 & 2.685 \\
9 & 11.200 & 0.447 & -0.057 & 0.353 & 2.684 \\
10 & 10.080 & 0.441 & -0.041 & 0.287 & 2.665 \\
11 & 10.880 & 0.531 & -0.114 & 0.612 & 2.740 \\
12 & 9.395 & 0.428 & -0.103 & 0.150 & 2.624 \\
16 & 11.635 & 0.504 & -0.097 & 0.680 & 2.725 \\
23 & 10.750 & 0.502 & -0.066 & 0.440 & 2.702 \\
26 & 9.647 & 0.482 & -0.110 & 0.174 & 2.625 \\
30 & 9.663 & 0.490 & -0.109 & 0.156 & 2.628 \\
35 & 10.453 & 0.436 & -0.071 & 0.270 & 2.656 \\
36 & 9.803 & 0.464 & -0.089 & 0.210 & 2.632 \\
37 & 9.650 & 0.440 & -0.074 & 0.177 & 2.625 \\
38 & 9.825 & 0.463 & -0.079 & 0.264 & 2.655 \\
40 & 11.155 & 0.446 & -0.086 & 0.421 & 2.691 \\
41 & 13.030 & 0.552 & +0.003 & 1.232 & 2.874 \\
42 & 12.590 & 0.510 & -0.078 & 0.960 & 2.770 \\
43 & 11.387 & 0.457 & -0.073 & 0.492 & 2.700 \\
44 & 10.800 & 0.498 & -0.084 & 0.385 & 2.701 \\
45 & 11.453 & 0.487 & -0.083 & 0.544 & 2.718 \\
49 & 10.707 & 0.452 & -0.073 & 0.319 & 2.667 \\
52 & 12.290 & 0.535 & -0.073 & 0.977 & 2.755 \\
55 & 7.940 & 0.023 & +0.189 & 0.952 & 2.908 \\
59 & 7.955 & 0.810 & +0.220 & 0.491 & 2.632 \\
60 & 9.615 & 0.216 & +0.138 & 0.878 & 2.814 \\
61 & 9.570 & 0.384 & -0.078 & 0.291 & 2.654 \\
78 & 10.935 & 0.424 & -0.073 & 0.454 & 2.683 \\
\hline & & & & &
\end{tabular}

and the dereddened $(b-y)_{0}$ and $c_{0}$ quantities (Eqs. (6)-(8)), and the calibration by Balona \& Shobbrook (1984) is used to derive the absolute magnitude $M_{V}$. For early spectral types the presence of stellar emission can be a significant source of error in the calculated $M_{V}$, but, as mentioned in the previous paragraph, this should not be an issue for our sample. In the case of emission-line stars, $\beta$ calculated from $c_{0}$ is often used. We compared the $M_{V}$ calculated from the observed $\beta$ and from $\beta\left(c_{0}\right)$ and, as expected for this sample, found no significant difference. In general, photometric $u v b y \beta$ parallaxes obtained via these calibrations have been shown to be in agreement with the HIPPARCOS parallaxes with small relative errors (Kaltcheva \& Golev 2011), and also with the Gaia data, according to our preliminary results.

It would be instructive to estimate how the application of various $M_{V}$ calibrations would affect the calculated $M_{V}$ for the stars of NGC 1502. We applied the Balona \& Shobbrook (1984) calibration to the 21 stars included in Table 3 of Crawford (1994b) and compared the result to the $M_{V}$ values obtained there. The Balona \& Shobbrook (1984) calibration provides an average $D M=9.86 \pm 0.42$ that agrees with the one by Crawford (1994b) of $9.71 \pm 0.41$. Although these two estimates are identical within the errors, the smaller latter value is due to the fact that the Crawford (1994b) calibration is based on the $\beta$ index only and, contrary to the Balona \& Shobbrook (1984) calibration, does not take into account the evolutionary effects. This leads to a slight underestimation ( $M_{V}$ less negative) of the absolute magnitude and thus of the distance. A similar conclusion was reached by Kaltcheva \& Knude (1998) via comparison of the results of the two calibrations to the HIPPARCOS data. However, in the case of NGC 1502 there is also a systematic trend between the two 
Table 8. $u v b y \beta$-based quantities of NGC 1502 early-type stars.

\begin{tabular}{|c|c|c|c|c|c|c|c|c|c|c|c|c|c|c|c|}
\hline Star & Sp Type & V & $b-y$ & $m_{1}$ & $c_{1}$ & $\beta$ & $E(b-y)$ & $(b-y)_{0}$ & $c_{0}$ & $M_{V}$ & $R$ & $A_{V}$ & $V_{0}$ & $D M$ & $d(\mathrm{pc})$ \\
\hline 1 & OIII & 6.925 & 430 & 0.138 & 054 & 2.57 & 0.550 & -0.120 & & $-4.55:$ & 2.79 & 2.08 & & .40: & 59: \\
\hline 2 & $\mathrm{O} 9 \mathrm{IV}+\mathrm{B} 0.5 \mathrm{~V}$ & & & & & & & & -0.039 & - & & & 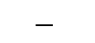 & & 977: \\
\hline 11 & $\mathrm{~B} 1.5 \mathrm{~V}$ & .880 & 531 & -0.114 & .612 & 2.740 & & 065 & 0.493 & & 2.96 & 2.38 & 8.50 & 8.87 & 594 \\
\hline 23 & B3V & .750 & 502 & -0.066 & .440 & 2.702 & & -0.082 & 0.323 & & 3.03 & 2.40 & 8.35 & 9.34 & 738 \\
\hline 44 & B2V & .800 & 498 & -0.084 & .385 & 2.701 & & 87 & 0.2 & -1.0 & 2.77 & 2.19 & 8.61 & 9.68 & 863 \\
\hline 55 & & & & & & 2.9 & & & & & 3.44 & & 7.74 & & 189 \\
\hline 61 & $\mathrm{~B} 2 \mathrm{~V}$ & 9.570 & 0.384 & -0.078 & 0.291 & 2.654 & 0.479 & & 0.19 & & 2.75 & 1.78 & 7.79 & 9.73 & 882 \\
\hline 7 & & & & -0.1 & & 2.6 & & & & & 2.76 & & 9.29 & & 1341 \\
\hline 10 & B & & & -0.0 & & 2.6 & & & & & 2.60 & & 8.19 & & 976 \\
\hline 12 & B2 & & 428 & -0.103 & 0.150 & 2.624 & & & & & 2.81 & 2.04 & 7.35 & 10.23 & 1113 \\
\hline 16 & B5V & 11.635 & 0.504 & -0.097 & 0.680 & 2.725 & & & & & 2.95 & 2.24 & 9.40 & 0.02 & 1009 \\
\hline 26 & B $1.5 \mathrm{~V}$ & 9.647 & 0.482 & -0.110 & 0.174 & 2.625 & 0.590 & -0.109 & 0.056 & -2.82 & 2.74 & 2.19 & 7.46 & 10.28 & 1140 \\
\hline 30 & B1V & 663 & 0.490 & -0.109 & 0.156 & 2.62 & 601 & .111 & 0.036 & -2.82 & 2.89 & 2.34 & 7.32 & 14 & 1067 \\
\hline 35 & B3V & 10.453 & 0.436 & -0.071 & 0.270 & 2.656 & & -0.098 & 0.163 & & 2.66 & 1.92 & 8.53 & 10.48 & 1245 \\
\hline 36 & B8V & 9.803 & 0.464 & -0.089 & 0.210 & 2.632 & & & & -2.56 & 2.58 & 1.98 & 7.82 & 10.38 & 1190 \\
\hline 37 & B $1.5 \mathrm{~V}$ & 9.650 & 0.440 & -0.074 & 0.177 & 2.625 & & -0.107 & 0.0 & -2.80 & 2.99 & 2.22 & 7.43 & & 1112 \\
\hline 40 & B2V & & 0.446 & -0.086 & 0.421 & 2.691 & & -0.083 & & & 2.67 & 1.91 & 9.25 & 10.42 & 1212 \\
\hline 42 & B9 & 12.590 & 0.510 & -0.078 & 0.960 & 2.770 & & -0.029 & & -0.17 & 2.86 & 2.08 & 10.51 & 10.68 & 1366 \\
\hline 43 & & 11.387 & 0.457 & -0.073 & 0.492 & 2.700 & & & & -0.99 & 2.83 & 2.03 & 9.35 & 10.35 & 1173 \\
\hline 45 & $\mathrm{~B} 4.5 \mathrm{~V}$ & 11.453 & 0.487 & -0.083 & 0.544 & 2.718 & & -0.071 & 0.4 & -0.70 & 2.97 & 2.24 & 9.21 & 9.92 & 962 \\
\hline 49 & B3V & & & -0.073 & & 2.667 & & & & & 2.91 & 2.15 & 8.56 & 10.24 & 1117 \\
\hline 52 & B9 & 12.290 & 0.535 & -0.073 & 0.977 & 2.755 & 0.563 & -0.028 & 0.864 & -0.44 & 3.06 & 2.32 & 9.97 & 10.40 & 1204 \\
\hline
\end{tabular}

Notes.The WEBDA identification, MK classification and homogenized $u v b y \beta$ photometry $V, b-y, m_{1}, c_{1}$, and $\beta$ are shown in the first part of the table. The color excesses $E(b-y)$ and selected dereddened quantities $(b-y)_{0}$ and $c_{0}$ are listed next, followed by the absolute magnitude $M_{V}$. The polarization-based values of $R$ and the extinction $A_{V}$ are included next, followed by the corrected apparent magnitude $V_{0}$, the true distance moduli and distance in parsecs.

sets of individual $M_{V}$ values and distance moduli which could prevent revealing details of the structure of the field. We note that the choice of the $M_{V}$ calibration is affecting the derived cluster parameters more significantly than the variations between different homogenization procedures of the photometric data.

As already mentioned, $u v b y \beta$-based cluster parameters of NGC 1502 have been obtained by several authors. The present work includes a number of improvements to these studies. First, we apply a better $M_{V}$ calibration. Second, the new polarimetric measurements are essential for the calculation of the actual total-to-selective extinction ratio $R$ for each star. This is expected to result in better distance determinations, thereby helping to identify foreground and background stars along the line of sight. The new homogenization of the existing photometry also adds to the analysis.

Table 8 contains selected photometry-derived parameters of the stars with available polarimetric measurements. Section 4.4 presents details on the adopted here distance moduli for the visual double star ADS 2984 (W1 and W2) that are listed at the beginning of the table. We omit the values for $M_{V}$ and $V_{0}$ for W2 from the table, since the primary and secondary components of SZ Cam proper are discussed in Sect. 4.4. Next, stars that appear as foreground are included, followed by the probable photometric members of NGC 1502. The WEBDA numbering system is shown in the first column, followed by the adopted spectral types and the homogenized $u v b y \beta$ photometry from Table 7 . The MK classification of W1 and W2 is discussed in Sect. 4.4. For the rest of the stars, we examined all available sources and gave preference to those providing both spectral type and luminosity class (Abt \& Cardona 1983; Hoag \& Applequist 1965). A sole source of homogeneous MK classification does not exists for our sample stars. The middle portion of the table includes the color excess $E(b-y)$, the dereddened $(b-y)_{0}$ and $c_{0}$ quantities and the calculated absolute magnitudes $M_{V}$. The last part contains $R=5.6 \lambda_{\max }, A_{V}=R E(b-y) / 0.74, V_{0}=V-A_{V}$, the true distance modulus $D M$ and the distance for each star. The uncertainties in $M_{V}$ based on the Balona \& Shobbrook (1984) calibration are of the order of $\pm 0.3 \mathrm{mag}$ for $\mathrm{O}$ and B III-V types, which propagates to an error of $-13 \%$ to $+15 \%$ in the derived distances.

Based on the data in Table 8, we plotted the color excess $E(b-y)$ vs. the photometric $D M$ for both $R=3.2$ and $R=$ $5.6 \lambda_{\max }$ (Fig. 9 top and middle panels). The dereddened $V_{0}$ versus $(b-y)_{0}$ diagram utilizing $R=5.6 \lambda_{\max }$ is shown on the bottom panel. Selected stellar isochrones based on the PARSEC evolutionary models for solar composition (Bressan et al. 2012) are overplotted on this diagram. The scatter in the distance modulus is relatively large, but no differential reddening is present. W55 is a nearby low-reddened star with $E(b-y)=0.04$. For the rest of the stars, the color excess $E(b-y)$ varies between 0.53 and 0.60 mag (20 stars), except for W61 (0.48 mag). Variation of this magnitude is probably too insignificant to imply differential reddening, moreover no obvious trend across the studied field of view is noticeable. In addition, there seems to be no difference in $P_{\max }$ across the field as well. The average color excess, based on these 20 stars is $E(b-y)=0.56 \pm 0.02$, which yields $A_{V}=2.42$ for $R=3.2$. The average polarization-based $R$ for the same stars is $2.83 \pm 0.14$, resulting in $A_{V}=2.14 \pm 0.16 \mathrm{mag}$.

We use different symbols to visualize possible foreground stars along the line of sight. Stars that are closer than the 3.2based $D M=9.5$ are marked with cross/plus symbols, and filled symbols are used for the more distant group. On all plots W1 

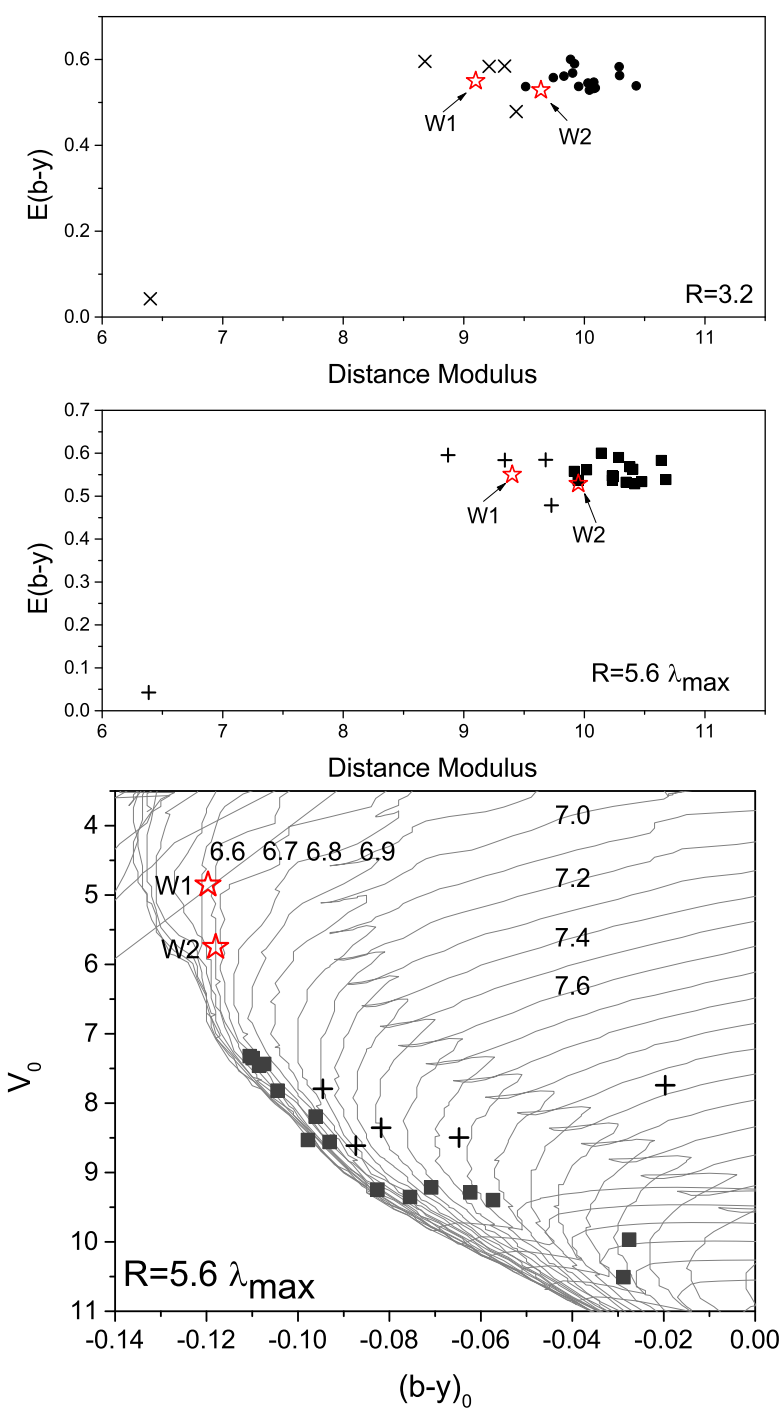

Fig. 9. Top and middle: color excess $E(b-y)$ vs. $D M$ for $R=3.2$ and $R=5.6 \lambda_{\max }$, respectively. Bottom: $V_{0}$ vs. $(b-y)_{0}$ diagram for $R=5.6 \lambda_{\max }$ with PARSEC isochrones (for solar composition) overplotted. Selected isochrones are labeled in terms of $\log t$. W1 and W2 are also labeled.

and $\mathrm{W} 2$ are labeled. The color-magnitude $(\mathrm{CM}) V_{0}$ vs. $(b-y)_{0}$ diagram reflects this separation as well. Apart of W55, other stars also appear to be foreground to the cluster and slightly more evolved on the $\mathrm{CM}$ diagram, at an average (polarizationbased) distance modulus $9.40 \pm 0.40(759 \mathrm{pc})$. If we use the polarization-based $R$, the 15 stars marked with filled symbols on the corresponding diagram are located between distance moduli 9.9 and 10.7, with an average $D M=10.29 \pm 0.22$. This is 0.3 mag larger in comparison to a $D M$ based on $R=3.2$ for the same 15 stars $(D M=10.00 \pm 0.23)$. If we exclude from the calculations the four fainter stars that deviate on the CM diagram from the MS defined by the brighter stars, the $D M$ does not change significantly: $D M=10.24 \pm 0.18$. Two of these stars (W42 and W52) have already been discussed as stars of spectral type B9, appearing more evolved on the $\beta$ vs. [ $\left.c_{1}\right]$ diagram. The other two stars (W7 and W16) also slightly deviate from the MS delineated by the rest of the stars on this diagram. Since there is a chance these fainter stars could be pre-main sequence candidates, we accept 10.24 as the true DM of NGC 1502 . Using this value to adjust the isochrones provides an age for this group of 6.7 in $\log t$ (5.01 Myr). This estimate is based on the brightest

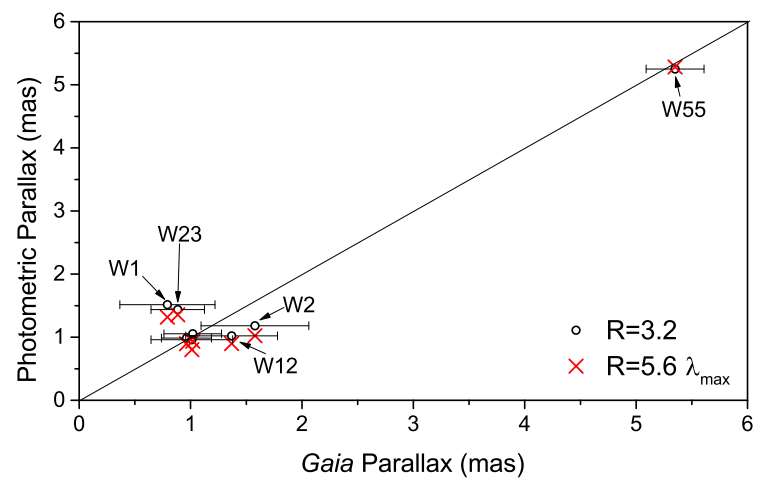

Fig. 10. Comparison between the photometric and Gaia parallaxes for $R=3.2$ and $R=5.6 \lambda_{\max }$.

stars ( $V_{0}$ around $7.5 \mathrm{mag}$ ). We do not include $\mathrm{W} 1$ and $\mathrm{W} 2$ in any of the above calculations because of their peculiar nature.

The comparison of the photometric to the currently available Gaia parallaxes (DR1) is shown on Fig. 10, both for $R=3.2$ and $R=5.6 \lambda_{\max }$. The errorbars for the photometric parallaxes are of the order of $15 \%$ of the parallax and since they are significantly smaller than the Gaia parallax errors, they are not plotted. Four stars that appear at the distance of NGC 1502 have Gaia parallaxes in DR1. The weighted mean parallax for them is $1.12 \pm 0.11$ mas, with a median value of 1.02 mas. This corresponds to an average distance of $892_{-79}^{+98} \mathrm{pc}$ and a median distance of $980_{-96}^{+118} \mathrm{pc}$. The $11 \mathrm{MS}$ cluster members discussed in the previous paragraph yield $968 \mathrm{pc}(D M=9.93)$ for $R=3.2$, and a polarization-based estimate of $1117_{-89}^{+96}(D M=10.24)$. Having in mind that the Gaia DR1 data are still preliminary, the $u v b y \beta$ distance obtained here adopting the polarization-based $R$ for each star should be considered the best current estimate for NGC 1502.

\subsection{Cluster parameters via $U B V$ photometry and $M K$ classification}

In addition to the $u v b y \beta$-based analysis we used the averaged $U B V$ photometry to infer color excesses and absolute magnitudes. In comparison to the sample of 22 stars considered in Sect. 4.2, Table 5 contains nine additional stars earlier than A0 type $\left((B-V)_{0}<0.0 \mathrm{mag}\right)$ that have $U B V$ data but not uvby $\beta$ and polarization data. For this larger sample we obtained color excesses via the Q-method and also the absolute magnitudes, applying both the formulas provided by Crawford (1994b) and Pecaut \& Mamajek (2013, PM13 hereafter). We note that the $M_{V}$ part of the PM13 calibration is provided only via the website maintained by E. Mamajek ${ }^{1}$. The PM13 calibration was applied to both $(B-V)_{0}$ and $(U-B)_{0}$ indices and the results for $M_{V}$ were averaged for each star, except for W1 and W2 which fall at the limit of the usable $(B-V)_{0}$ calibration range. These $M_{V}$ estimates may be uncertain for W2 and W2 also due to uncertainties in luminosity class. Table 9 lists the dereddened $U B V$ quantities, $E(B-V), M_{V}$ and $D M$ obtained following PM13.

The top panel of Fig. 11 presents a comparison of the two sets of $U B V$-based magnitudes derived here following Crawford (1994b) and PM13. This plot also includes the $u v b y \beta$-based $M_{V}$ values listed in Table 8 and spectroscopic $M_{V}$ based on the MK classification from Table 5 and the PM13 calibration via MK.

\footnotetext{
1 www.pas.rochester.edu/ emamajek/EEM_dwarf_UBVIJHK_ colors_Teff.txt
} 
Table 9. Dereddened $U B V$ photometry $(U-B)_{0}$ and $(B-V)_{0}$, color excess $E(B-V)$, absolute magnitudes $M_{V}$ and true distance moduli $D M$ for stars toward NGC 1502.

\begin{tabular}{cccccc}
\hline \hline Star & $(U-B)_{0}$ & $(B-V)_{0}$ & $E(B-V)$ & $M_{V}$ & $D M$ \\
\hline 1 & -1.07 & -0.30 & 0.77 & $-3.92::$ & $8.74::$ \\
2 & -1.05 & -0.29 & 0.71 & $-3.71::$ & - \\
7 & -0.50 & -0.14 & 0.72 & -0.49 & 10.03 \\
10 & -0.78 & -0.21 & 0.78 & -1.79 & 9.57 \\
11 & -0.60 & -0.16 & 0.79 & -0.88 & 9.42 \\
12 & -0.94 & -0.26 & 0.73 & -2.80 & 10.16 \\
13 & -0.38 & -0.11 & 0.67 & -0.09 & 10.95 \\
16 & -0.56 & -0.15 & 0.76 & -0.70 & 10.08 \\
17 & -0.19 & -0.07 & 0.81 & 0.53 & 10.75 \\
18 & -0.06 & -0.03 & 0.84 & 0.97 & 10.50 \\
23 & -0.69 & -0.19 & 0.81 & -1.28 & 9.56 \\
24 & -0.37 & -0.11 & 0.83 & -0.03 & 10.45 \\
26 & -0.93 & -0.26 & 0.82 & -2.72 & 10.10 \\
27 & -0.59 & -0.16 & 0.71 & -0.83 & 11.27 \\
30 & -0.98 & -0.27 & 0.85 & -3.10 & 10.31 \\
35 & -0.82 & -0.22 & 0.74 & -2.00 & 10.53 \\
36 & -0.91 & -0.25 & 0.81 & -2.55 & 10.25 \\
37 & -0.79 & -0.21 & 0.70 & -1.80 & 8.99 \\
40 & -0.68 & -0.18 & 0.70 & -1.23 & 10.65 \\
41 & -0.12 & -0.04 & 0.88 & 0.78 & 9.65 \\
42 & -0.29 & -0.09 & 0.72 & 0.22 & 10.28 \\
43 & -0.61 & -0.16 & 0.72 & -0.92 & 10.25 \\
44 & -0.78 & -0.21 & 0.81 & -1.76 & 10.32 \\
45 & -0.62 & -0.17 & 0.79 & -0.95 & 10.03 \\
49 & -0.77 & -0.21 & 0.73 & -1.68 & 10.27 \\
52 & -0.07 & -0.03 & 0.73 & 0.92 & 9.10 \\
55 & -0.01 & -0.01 & 0.06 & 1.15 & 6.57 \\
60 & -0.12 & -0.05 & 0.37 & 0.75 & 7.86 \\
61 & -0.83 & -0.23 & 0.66 & -2.04 & 9.78 \\
76 & -0.84 & -0.23 & 0.81 & -2.15 & 10.24 \\
78 & -0.69 & -0.18 & 0.69 & -1.28 & 10.25 \\
\hline & & & & & \\
\hline
\end{tabular}

$\mathrm{W} 1$ and W2 are excluded from the first two diagrams on Fig. 11. Despite for several deviating stars, the best agreement is between the $M_{V}$ values derived via the PM13 calibration and the $u v b y \beta$ values (for an intercept fixed at 0.0 , the slope is $1.055 \pm 0.059$ ). The scatter in the spectroscopic $M_{V}$ values is somehow larger. This is very possibly due to uncertainties in the adopted spectral classification, in addition to the fact that the spectroscopic absolute magnitude has mostly a statistical meaning. The $U B V$-based absolute magnitudes obtained following Crawford (1994b) show a small nonlinear dependence when compared to the results of the rest of the calibrations, which is expected in the light of the discussion in Sect. 4.2.

The $E(B-V)$ vs. $D M$ diagram utilizing the PM13 calibration is shown on the second panel of Fig. 11. For the nine stars without polarimetric measurements we adopted the average for the cluster $R=2.83$. These $U B V$-based determinations are plotted with filled squares. For completeness we included in the diagram the $u v b y \beta$ values from Table 8 (plus symbols), using the relation $E(b-y)=0.74 E(B-V)$. The spectroscopic determinations are shown with open squares. All three datasets reveal a relatively tight clustering between $D M$ 9.7 and $10.7 \mathrm{mag}$ and some foreground stars. Based on 18 stars with $U B V$ determinations located in this distance range, we calculated an average $D M=10.30 \pm 0.21$ for the cluster (median value $10.26 \mathrm{mag}$ ). The agreement between this estimate
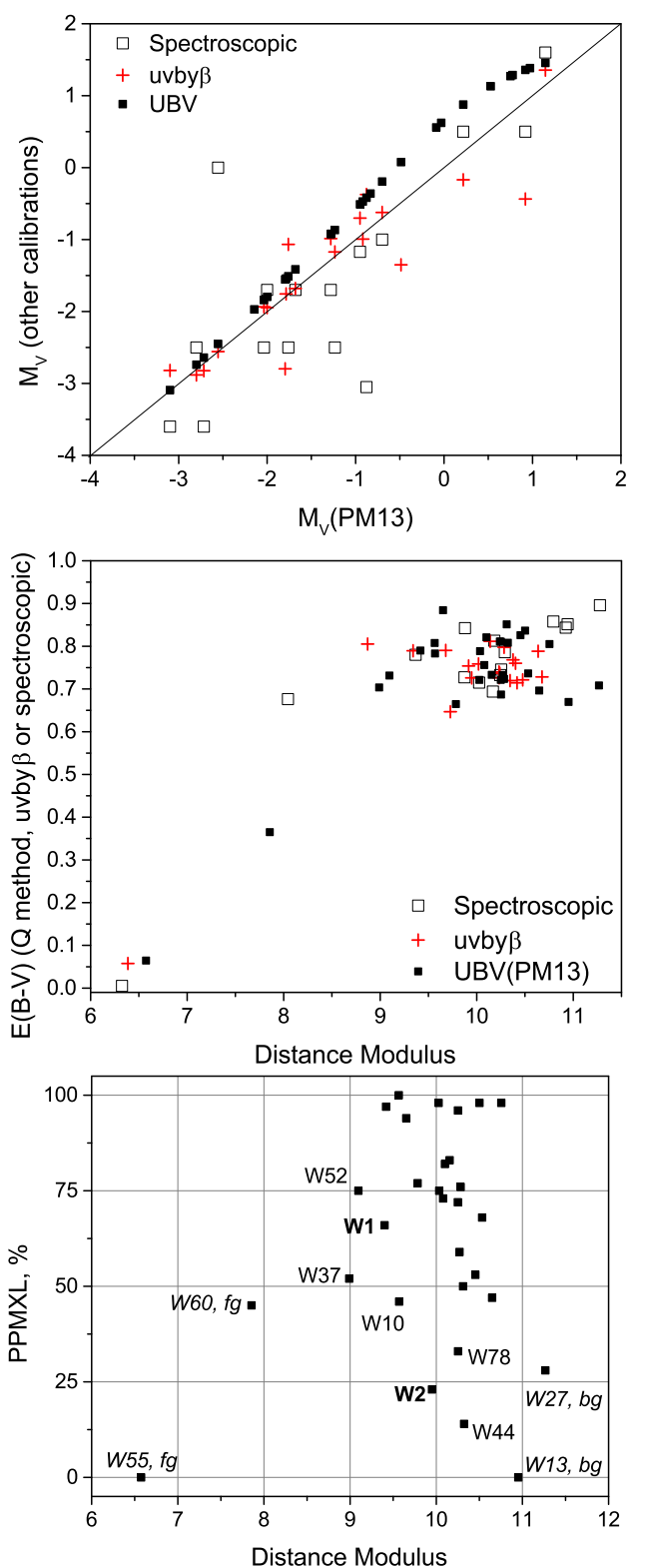

Fig. 11. Top: comparison of spectroscopic, $u v b y \beta$ and $U B V$ (Crawford 1994b) values to the $M_{V}$ values obtained via the calibration of Pecaut \& Mamajek (2013). Middle: color excess vs. distance modulus diagram emphasizing the $U B V$-based results. Bottom: membership probabilities vs. the $U B V$-based distance moduli.

and the one based on $u v b y \beta$ photometry is excellent. There is also good agreement between the $U B V$ and $u v b y \beta$-based color excesses, indicating no differential reddening. The $u v b y \beta$-based values show slightly less scatter which could be contributed to the higher precision in the physical parameters derivation, as expected for an intermediate-band photometry.

Figure 11, bottom panel, presents the individual membership probabilities obtained from the proper motions of the PPMXL (Roeser et al. 2010) vs. DM based on PM13. The derivation of the individual membership probabilities is described in detail in Sect. 3. This plot is constructed utilizing the larger sample with available $U B V$ photometry. It is consistent with a similar plot based on the $u v b y \beta$ data. For W1 and W2 distance moduli of 9.40 and $9.95 \mathrm{mag}$, respectively, are utilized based on the analysis in Sect. 4.4. This places W1 at the near end of the main concentration and W2 within the cluster. As expected, 
the four stars that are photometrically foreground (marked as $f g$ ) and background (marked as $b g$ ) have lower membership probabilities according to most of the estimates. Excluding several stars that have borderline distance moduli ( 9 to 9.5 ) or membership probabilities (around 50\%) leaves a group at a distance modulus of about 10.25 mag that is identified with the cluster. We note that $\mathrm{W} 37$ is a variable star and the $D M$ based on $U B V$ is possibly underestimated by 0.4 mag (mostly due to the accepted $V$-magnitude). For the rest of the deviating stars (W44, W52, and W78) the individual membership probabilities based on PPMXL and UCAC5 are quite different, and that uncertainty may contribute to the scatter. In general, we notice the good correspondence between the PPMXL probabilities and the derived individual distance moduli.

\subsection{ADS 2984}

The two brightest stars in the field, W1 and W2, form the visual double star ADS 2984. The spectroscopic binary ADS 2984A (W1, HD 25639) has been classified in the literature as B0 II-III (Morgan et al. 1955, Münch 1957, Meadows 1961, Walker 1963, Harris 1976), B0IIn (Abt 1981), B0 III (Lesh 1968), O9 V (Murphy 1969) or O9 III (Lutz \& Lutz 1977). The $V$ mag is 6.86 in SIMBAD and between 7.1 and 7.15 in Kukarkin et al. (1981). No light variations within 0.01 mag have been recorded for ADS 2984A (see Gorda 2016 for details). Lorenz et al. (1998) estimated the contribution of the secondary component as less than 5\%. They found the primary component to be relatively fast rotating $\left(220 \pm 20 \mathrm{~km} \mathrm{~s}^{-1}\right)$ and suggested a slightly later spectral type than the SZ Cam primary. For the accepted here $V=6.925$ the $u v b y \beta$ distance modulus is 9.4 (759 pc) for the polarization-based $A_{V}=2.08$ (Table 8). A spectroscopic distance would depend on the accepted classification. A classification B0III yields $M_{V}=-5.0 \mathrm{mag}$ (Deutschman et al. 1976), while the $U B V$-based $M_{V}$ of -3.92 mag is probably underestimated due to uncertainties of the calibration at the high-mass end. In the case of $\mathrm{W} 1$, the $u v b y \beta$-based $M_{V}=-4.55$ mag seems the most reliable estimate and we accept this value to obtain the distance, as listed in Table 8 . W1 was recently studied by Gorda (2015) who obtained 0.4 for the mass ratio of the components. This mass ratio justifies a correction of about $0.3 \mathrm{mag}$ to the visual magnitude (assuming equal surface temperatures and a mass-radius relation in the form $R \sim M^{0.555}$, Demircan \& Kahraman 1991). With the correction, this distance becomes 869 pc $(D M=9.695)$ which sets the upper limit of the distance estimate possible via this homogenized $u v b y \beta$ data. However, a correction of this magnitude is not entirely consistent with the study by Lorenz et al. (1998) and we did not consider it further. The TGAS Gaia-based distance is uncertain $\left(1263_{-443}^{+1477} \mathrm{pc}\right)$.

ADS 2984B (W2, SZ Cam, HD 25638) is a quadruple system consisting of two close binaries in a relative orbit with a period of approximately $50 \mathrm{yr}$ (Gorda 2015). The MK classification existing in the literature, has not been entirely consistent, especially regarding the luminosity class, varying from III to $\mathrm{V}$ : O9V, O9.5Vn, B0V (Murphy 1969, Lutz \& Lutz 1977), B1III (Harris 1976). The structure and the components of W2 have been discussed in detail by several authors (see Gorda 2015 and the references therein). The eclipsing (also spectroscopic binary) pair, SZ Cam proper, consists of early-type high-mass components presently classified as O9IV+B0.5V (Lorenz et al. 1998). The light contribution of the third body in the ADS 2984B system has been estimated as $30 \%$ by speckle interferometry (Mason et al. 1998, Balega et al. 2007). The most recent estimate of the mass ratio of the SZ Cam proper components, $0.72 \pm 0.01$, is in agreement with the majority of the previous estimates (Gorda 2015).

Budding (1975) first classified the primary as O9.5nk (V) and obtained a distance of $600 \pm 150 \mathrm{pc}$. Based on their light-curve solution, Lorenz et al. (1998) calculated a photometric distance moduli of $9.70 \mathrm{mag}(871 \mathrm{pc})$ and $9.61 \mathrm{mag}(836 \mathrm{pc})$ for the primary and the secondary component, using $A_{V}=2.32$, in good agreement with the cluster distance as obtained by Crawford (1994b). If we adopt the polarization-based $A_{V}=1.98$ for W2 obtained here, these distances become slightly larger, 1019 pc and $977 \mathrm{pc}$, respectively, associating, within the errors, W2 with the NGC 1502 cluster at $1.1 \mathrm{kpc}$.

Hilditch \& Hill (1975) observed SZ Cam in uvby outside eclipses and obtained averaged quantities $V=6.94$, $b-y=0.431, m_{1}=-0.107$ and $c_{1}=0.002$. These data have not been implemented in any of the existing catalogs, including our homogenization. The assumption that the third-light contribution to the apparent visual magnitude of $\mathrm{W} 2$ is $30 \%$ yields a total visual magnitude of $V=7.33$ mag for SZ Cam proper. Using a mass-ratio of 0.72 for the primary and secondary components, and a mass-radius relation in the form $R \sim M^{0.555}$, we obtained apparent magnitudes of 7.73 and $8.75 \mathrm{mag}$, respectively. In these calculations we used the temperatures of the primary and secondary as $32500 \mathrm{~K}(\mathrm{O} 9 \mathrm{~V})$ and $29000 \mathrm{~K}(\mathrm{~B} 0.5 \mathrm{~V})$, respectively, based on the PM13 calibration. The obtained here $A_{V}=1.98$ and spectroscopic absolute magnitudes -4.2 and -3.6 , yield a slightly different distance moduli of 9.95 and 10.73 mag for the two components of SZ Cam prior. However, accounting for the different $M_{V}$ calibrations and mass-ratios used, this is in agreement with Lorenz et al. (1998).

We note that since the eclipsing pair consists of stars of very similar spectral types, the photometric colors should provide a reasonable estimate for the extinction and also for the absolute magnitude of the intrinsically brighter component. However, the $U B V$-based $M_{V}=-3.71 \mathrm{mag}$ might be underestimated due to the uncertainty in the $M_{V}$ determination for stars more massive than spectral type B0. The "outside eclipses" uvby photometry (Hilditch \& Hill 1975) yields $M_{V}=-4.46$ for $\beta=2.591$, and the new homogenization provides $M_{V}=-4.04$. Harries et al. (1998) obtained $M_{V}=-4.16$ for the primary component for a mass-ratio of 0.87. Based on these estimates, it seems justified to accept $M_{V}=-4.2 \mathrm{mag}$ for the primary component, which results in $D M=9.95 \mathrm{mag}(977 \mathrm{pc})$. These are the values we list in Table 8. Although the error is difficult to evaluate, this distance seems consistent with the distance for the cluster of $1117_{-89}^{+96}$ pc obtained here. However, the TGAS Gaia-based distance of $634_{-149}^{+280} \mathrm{pc}$ and the membership probabilities are not too supportive for membership of W2 to the cluster. Previous distance determinations have been summarized by Hohle et al. (2009) and vary between 600 and 1050 pc. The second Gaia data release is expected to provide more conclusive data for both W1 and $\mathrm{W} 2$.

\section{Concluding remarks}

Using new homogenization of the existing $u v b y \beta$ photometries and values of the total-to-selective extinction obtained via multiwavelength polarization observations, we present improved parameters of the young open cluster NGC 1502. Neither photometry nor polarization indicate differential reddening for the studied field. The average polarization-based total-to-selective extinction ratio toward the cluster obtained here is $R=2.83$. This is lower than the Galactic average and yields a visual absorption 
$A_{V}=2.14$ mag for the studied field of view. The uniformity of the polarization parameters across the cluster and the distribution of the color excess with the distance suggest that the extinction originates in the foreground of the cluster, between 200 and $600 \mathrm{pc}$, where the stars that appear as foreground are located. The dust causing the polarization is also located within this distance range. A larger sample of field stars is needed to map the dust distribution in more detail. However, such a sample with existing optical photometry is not available at present. In addition, the investigation by Green et al. (2015) also indicates lack of foreground stars in the direction of the field of view studied here.

We use 11 probable photometric members of NGC 1502 that all appear to be main sequence stars to obtain the cluster's distance as $1117_{-89}^{+96} \mathrm{pc}$. The $1-\sigma$ error of this determination is less than $9 \%$. This distance is identical to the one we obtain via the averaged $U B V$ photometry of 18 probable photometric members. In general, the individual kinematic membership probabilities derived here based on PPMXL support the selection of members based on photometric distances. This polarizationbased distance is about $150 \mathrm{pc}(15 \%)$ farther than estimates based on the galactic average $R=3.2$. Under the assumption of solar metallicity, the cluster is $5 \mathrm{Myr}$ young according to the PARSEC isochrones. At this point, it is difficult and probably not meaningful to attempt a detailed comparisons of the results obtained here to previous photometric studies because of different absolute magnitude calibrations, values of $R$ and evolutionary models used. The suggested new distance of $1.1 \mathrm{kpc}$ is well within the range of the previous estimates. The derived age of $5 \mathrm{Myr}$ is consistent with the deviating position on the CM diagram of some of the fainter probable members of the cluster that could possibly indicate a pre-main sequence status, thus a very young cluster. The result on a value of $R$ lower than the galactic average seems robust. It is based on the larger sample presently available of probable cluster members, and supports and refines previous similar findings.

The adopted here photometric distance of SZ Cam (W2) is consistent with the average distance of the cluster, while HD 25639 (W1) may be foreground. Since both stars are known as double-lined spectroscopic binaries, any photometric distance should be considered with caution. From another standpoint, several sources estimate a low individual membership probability of W2. Future Gaia data releases would provide more clarity on the membership of HD 25639 and SZ Cam to the cluster and more detail on the foreground stellar population in this direction.

Acknowledgments. This work is supported by a Jackson-Hope Foundation Grant-in-aid of research at the Virginia Military Institute and by an NSF grant AST-1516932 and sabbatical award FDS507 at the University of Wisconsin Oshkosh. This work has made use of data from the European Space Agency (ESA) mission Gaia (http://www.cosmos.esa.int/gaia), processed by the Gaia Data Processing and Analysis Consortium (DPAC, http://www. cosmos. esa.int/web/gaia/dpac/consortium). Funding for the DPAC has been provided by national institution, in particular the institution participating in the Gaia Multilateral Agreement. This study made use of the NASA Astrophysics Data System, SIMBAD database, Centre de Données Stellaires (http://cdsweb.u-strasbg.fr/) and the WEBDA open cluster database operated at the Department of Theoretical Physics and Astrophysics of the Masaryk University (http://www.univie.ac.at/webda/ or http:// webda.physics.muni.cz). We are thankful to the referee for many excellent recommendations that helped to improve this paper.

\section{References}

Aannestad, P. A. 1982, A\&A, 115, 219

Aannestad, P. A., \& Greenberg, J. M. 1983, ApJ, 272, 551

Abt, H. A. 1981, ApJS, 45, 437

Abt, H. A., \& Cardona, O. 1983, ApJ, 272, 182
Altmann, M., Roeser, S., Demleitner, M., Bastian, U., \& Schilbach, E. 2017, A\&A, 600, L4

Alves, V. M., Pavani, D. B., Kerber, L. O., \& Bica, E. 2012, New Astron., 17, 488 Appenzeller, I. 1968, ApJ, 151, 907

Balaguer-Núnez, L., Tian, K. P., \& Zhao, J. L. 1998, A\&AS, 133, 387

Balega, I. I., Balega, Y. Y., Maksimov, A. F., et al. 2007, Astrophys. Bull., 62, 339

Balona, L. A., \& Shobbrook, R. R. 1984, MNRAS, 211, 375

Bressan, A., Marigo, P., Girardi, L., et al. 2012, MNRAS, 427, 127

Budding, E. 1975, Ap\&SS, 36, 329

Clarke, D. 2010, Stellar Polarimetry (Weinheim: Wiley-VCH Verlag GmbH \& Co. $\mathrm{KGaA}$ )

Clarke, D., \& Al-Roubaie, A. 1983, MNRAS, 202, 173

Clarke, D., \& Al-Roubaie, A. 1984, MNRAS, 206, 729

Clayton, G. C., Wolff, M. J., Sofia, U. J., Gordon, K. D., \& Misselt, K. A. 2003, ApJ, 588, 871

Crawford, D. L. 1975, AJ, 80, 955

Crawford, D. L. 1978, AJ, 83, 48

Crawford, D. L. 1994a, Mex. Astron. Astrofis., 29, 115

Crawford, D. L. 1994b, PASP, 106, 397

Crawford, D. L. 1999, in Precision CCD Photometry, eds. E. R. Craine, D. L.

Crawford, \& R. A. Tucker, ASP Conf. Ser., 189, 9

Crawford, D. L., Barnes, J. V., \& Golson, J. C. 1971, AJ, 76, 1058

Crézé, M. 1972, A\&A, 21, 85

Delgado, A. J., Alfaro, E. J., Garcia-Pelayo, J. M., \& Garrido, R. 1992, AJ, 103, 891

Demircan, O., \& Kahraman, G. 1991, Ap\&SS, 181, 313

Deutschman, W. A., Davis, R. J., \& Schild, R. E. 1976, ApJS, 30, 97

Dias, W. S., Monteiro, H., Caetano, T. C., et al. 2014, A\&A, 564, A79

di Serego Alighieri, S. 1997, in Instrumentation for Large Telescopes, eds. J. M. Rodríguez Espinosa, A. Herrero, \& F. Sánchez, 287

Dombrovskii, V. L., \& Hagen-Thorn, V. A. 1964, Trudy Astronomicheskoj Observatorii Leningrad, 20, 75

Eggen, O. J. 1968, Three-colour Photometry of 4000 Northern Stars (London: H.M.S.O.)

Gaia Collaboration 2016, VizieR Online Data Catalog: I/337

Gorda, S. Y. 2015, Astron. Lett., 41, 276

Gorda, S. Y. 2016, Astron. Lett. 42, 693

Green, G. M., Schlafly, E. F., Finkbeiner, D. P., et al. 2015, ApJ, 810, 25

Guetter, H. H. 1964, Publications of the David Dunlap Observatory, 2, 405

Handler, G. 2011, A\&A, 528, A148

Harries, T. J., Hilditch, R. W., \& Hill, G. 1998, MNRAS, 295, 386

Harris, G. L. H. 1976, ApJS, 30, 451

Hauck, B., \& Mermilliod, M. 1998, A\&AS, 129, 431

Hilditch, R. W., \& Hill, G. 1975, Mem. R. Astron. Soc., 79, 101

Hiltner, W. A. 1951, ApJ, 114, 241

Hoag, A. A. 1953, AJ, 58, 42

Hoag, A. A., \& Applequist, N. L. 1965, ApJS, 12, 215

Hoag, A. A., Johnson, H. L., Iriarte, B., et al. 1961, Publications of the U.S. Naval Observatory Second Series, 17, 344

Hohle, M. M., Eisenbeiss, T., Mugrauer, M., et al. 2009, Astron. Nachr., 330, 511

Hsu, J.-C., \& Breger, M. 1982, ApJ, 262, 732

Javakhishvili, G., Kukhianidze, V., Todua, M., \& Inasaridze, R. 2006, A\&A, 447, 915

Johnson, H. L., Hoag, A. A., Iriarte, B., Mitchell, R. I., \& Hallam, K. L. 1961, Lowell Observatory Bulletin, 5, 133

Kaltcheva, N., \& Golev, V. 2011, in Stellar Clusters and Associations: A RIA Workshop on Gaia, 299

Kaltcheva, N., \& Knude, J. 1998, A\&A, 337, 178

Kharchenko, N. V., Piskunov, A. E., Schilbach, E., Röser, S., \& Scholz, R.-D. 2013, A\&A, 558, A53

Kitamura, M., \& Yamasaki, A. 1972, Tokyo Astron. Bull., 220

Kukarkin, B. V., Kholopov, P. N., Artiukhina, N. M., et al. 1981, Nachrichtenblatt der Vereinigung der Sternfreunde

Lesh, J. R. 1968, ApJS, 17, 371

Lorenz, R., Mayer, P., \& Drechsel, H. 1998, A\&A, 332, 909

Lutz, T. E., \& Lutz, J. H. 1977, AJ, 82, 431

Mason, B. D., Gies, D. R., Hartkopf, W. I., et al. 1998, AJ, 115, 821

Mathis, J. S., Rumpl, W., \& Nordsieck, K. H. 1977, ApJ, 217, 425

Meadows, A. J. 1961, MNRAS, 123, 81

Morgan, W. W., Code, A. D., \& Whitford, A. E. 1955, ApJS, 2, 41

Münch, G. 1957, ApJ, 125, 42

Murphy, R. E. 1969, AJ, 74, 1082

Netopil, M., Paunzen, E., \& Carraro, G. 2015, A\&A, 582, A19

Pandey, A. K., Upadhyay, K., Nakada, Y., \& Ogura, K. 2003, A\&A, 397, 191

Paunzen, E. 2015, A\&A, 580, A23

Paunzen, E., Netopil, M., Iliev, I. K., et al. 2005, A\&A, 443, 157

Pecaut, M. J., \& Mamajek, E. E. 2013, ApJS, 208, 9 
Planck Collaboration Int. XIX. 2015, A\&A, 576, A104

Purgathofer, A. 1961, Z. Astrophys., 52, 186

Purgathofer, A. 1964, Annalen der K.K. Sternwarte Wien, 26, 37

Reimann, H.-G., \& Pfau, W. 1987, Astron. Nachr., 308, 111

Roeser, S., Demleitner, M., \& Schilbach, E. 2010, AJ, 139, 2440

Schmidt, E. G. 1984, ApJS, 55, 455

Serkowski, K. 1974, in Planets, Stars, and Nebulae: Studied with Photopolarimetry, ed. T. Gehrels (Tucson: University of Arizona Press), IAU Colloq. 23, 135

Serkowski, K., Mathewson, D. S., \& Ford, V. L. 1975, ApJ, 196, 261

Shaw, J. S. 1975, A\&A, 41, 367

Simmons, J. F. L., \& Stewart, B. G. 1985, A\&A, 142, 100

Skrutskie, M. F., Cutri, R. M., Stiening, R., et al. 2006, AJ, 131, 1163

Straižys, V. 1992, Multicolor Stellar Photometry (Tucson: Pachart Publ. House)

Strömgren, B. 1966, ARA\&A, 4, 433

Tapia, M., Costero, R., Echevarria, J., \& Roth, M. 1991, MNRAS, 253, 649

Topasna, G. A., Topasna, D. M., \& Popko, G. B. 2013, PASP, 125, 1056

Topasna, G. A., Daman, E. A., \& Kaltcheva, N. T. 2017, PASP, 129, 104201
Tripathi, A., Pandey, U. S., \& Kumar, B. 2013, Bull. Astron. Soc. India, 41, 209

Walker, G. A. H. 1963, MNRAS, 125, 141

Wardle, J. F. C., \& Kronberg, P. P. 1974, ApJ, 194, 249

Weitenbeck, A. J. 1997, AJ, 114, 222

Weitenbeck, A. J. 2004, Acta Astron., 54, 87

Weitenbeck, A. J., Anderson, C. M., Code, A. D., WUPPE Team, \& Guest Investigators 1996, in Polarimetry of the Interstellar Medium, eds. W. G. Roberge, \& D. C. B. Whittet, ASP Conf. Ser., 97, 183

Weitenbeck, A. J., Halstead, E. A., \& Carver, A. J. 2008, Acta Astron., 58, 41

Whittet, D. C. B. 1977, MNRAS, 180, 29

Whittet, D. C. B. 2003, Dust in the Galactic Environment (Bristol: Institute of Physics)

Whittet, D. C. B. 2004, in Astrophysics of Dust, eds. A. N. Witt, G. C. Clayton, \& B. T. Draine, ASP Conf. Ser., 309, 65

Wilking, B. A., Lebofsky, M. J., \& Rieke, G. H. 1982, AJ, 87, 695

Zacharias, N., Finch, C. T., Girard, T. M., et al. 2013, AJ, 145, 44

Zacharias, N., Finch, C., \& Frouard, J. 2017, AJ, 153, 166

Zejda, M., Paunzen, E., Baumann, B., Mikulášek, Z., \& Liška, J. 2012, A\&A, 548, A97 